\title{
Effects of High-Intensity Interval Training on Aerobic Capacity in Cardiac Patients: A Systematic Review with Meta-Analysis
}

\author{
Bin Xie, ${ }^{1}$ Xianfeng Yan, ${ }^{1}$ Xiangna Cai, ${ }^{2}$ and Jilin $\mathrm{Li}^{1}$ \\ ${ }^{1}$ Department of Cardiology, First Affiliated Hospital of Shantou University Medical College, Shantou, Guangdong 515041, China \\ ${ }^{2}$ Department of Plastic Surgery, First Affiliated Hospital of Shantou University Medical College, Shantou, Guangdong 515041, China \\ Correspondence should be addressed to Xiangna Cai; caixiangna@126.com and Jilin Li; 1ijilin@126.com
}

Received 22 October 2016; Revised 7 January 2017; Accepted 19 January 2017; Published 12 March 2017

Academic Editor: Nikolaos G. Koulouris

Copyright (c) 2017 Bin Xie et al. This is an open access article distributed under the Creative Commons Attribution License, which permits unrestricted use, distribution, and reproduction in any medium, provided the original work is properly cited.

\begin{abstract}
Purpose. The aim of this study was to compare the effects of high-intensity interval training (INTERVAL) and moderate-intensity continuous training (CONTINUOUS) on aerobic capacity in cardiac patients. Methods. A meta-analysis identified by searching the PubMed, Cochrane Library, EMBASE, and Web of Science databases from inception through December 2016 compared the effects of INTERVAL and CONTINUOUS among cardiac patients. Results. Twenty-one studies involving 736 participants with cardiac diseases were included. Compared with CONTINUOUS, INTERVAL was associated with greater improvement in peak $\mathrm{VO}_{2}$ (mean difference $1.76 \mathrm{~mL} / \mathrm{kg} / \mathrm{min}, 95 \%$ confidence interval 1.06 to $2.46 \mathrm{~mL} / \mathrm{kg} / \mathrm{min}, p<0.001$ ) and $\mathrm{VO}_{2}$ at AT (mean difference $0.90 \mathrm{~mL} / \mathrm{kg} / \mathrm{min}, 95 \%$ confidence interval 0.0 to $1.72 \mathrm{~mL} / \mathrm{kg} / \mathrm{min}, p=0.03$ ). No significant difference between the INTERVAL and CONTINUOUS groups was observed in terms of peak heart rate, peak minute ventilation, $\mathrm{VE} / \mathrm{VCO}_{2}$ slope and respiratory exchange ratio, body mass, systolic or diastolic blood pressure, triglyceride or low- or high-density lipoprotein cholesterol level, flow-mediated dilation, or left ventricular ejection fraction. Conclusions. This study showed that INTERVAL improves aerobic capacity more effectively than does CONTINUOUS in cardiac patients. Further studies with larger samples are needed to confirm our observations.
\end{abstract}

\section{Introduction}

Cardiovascular diseases (CVDs) remain the greatest cause of death worldwide. In 2008, more than 17 million people died due to CVDs, of whom 7.3 million died of heart attacks [1]. Interventions are urgently needed to address this worrying trend. CVDs are largely preventable, and cardiac rehabilitation is increasingly recognized as an important component of the continuum of care for patients with coronary artery disease (CAD) and chronic heart failure (CHF). It is included in Class 1 recommendations of the American Heart Association and the American College of Cardiology for the treatment of these patients $[2,3]$.

According to the World Health Organization, insufficient physical activity is the fourth leading risk factor for mortality, with $6 \%$ of deaths worldwide attributed to this factor [1]. Exercise training is essential for cardiac patients. It has an important role in improving endothelial function, which in turn enhances blood flow by causing vasodilatation and improving vasomotor function. Exercise training also contributes to the improvement of many other functions, such as the achievement of good glycemic control and insulin sensitivity, leading to weight loss; the improvement of blood pressure; and the correction of deranged lipid profiles $[4,5]$. Proper exercise training is a cost-effective and well-established primary intervention that delays the onset of health burdens associated with various chronic diseases in many cases. The appropriate amount, frequency, and mode of exercise, however, remain unknown. Moreover, the optimum "dose" of exercise to obtain maximum cardiac benefits remains unclear.

Aerobic capacity has been found to be the single best parameter of cardiac function and all-cause death among known cases of CVDs [6]. It is measured directly as peak $\mathrm{VO}_{2}$. The improvement of the peak $\mathrm{VO}_{2}$ can improve aerobic capacity and promote cardiac rehabilitation. Moreover, 
reduction of the most common traditional risk factors for CVDs (e.g., hypertension, hyperlipidemia, and obesity) can decrease the occurrence of cardiovascular events. Research suggests that CAD and CHF are associated with impaired endothelial dysfunction, which is evaluated by flow-mediated dilation (FMD) and can be improved through physical exercise [7]. Thus, the identification of more effective exercise programs is needed to improve cardiovascular benefits in cardiac patients.

Moderate-intensity continuous training (CONTINUOUS), a traditional exercise prescription, usually involves walking or cycling for 30-60 min [to reach $40-80 \%$ peak oxygen uptake (peak $\mathrm{VO}_{2}$ )] [8]. However, recent evidence from patients with CHF [9] and CAD [10] suggests that high-intensity interval training (INTERVAL) may be a better modality for the improvement of aerobic capacity. Although INTERVAL has no standard definition, it refers to repeated sessions of brief intermittent exercise, often performed with maximal effort or intensity (i.e., to achieve $\geq 90 \%$ peak $\mathrm{VO}_{2}$ ) [11]. This intensity can be achieved by a single effort lasting a few seconds to several minutes, or with multiple efforts separated by a few minutes of rest or low-intensity exercise. INTERVAL has been shown to have significant benefits, including improved aerobic capacity, endothelial function, and other cardiac functions, in patients with CAD and CHF $[12,13]$.

Although several reviews and meta-analyses of INTERVAL for CAD and CHF were published [14-16], no consensus has been reached about whether INTERVAL produces superior physical, clinical, and functional benefits compared to CONTINUOUS. We are also unware of any systematic reviews that have assessed the effect of INTERVAL among cardiac patients.

This systematic review was conducted to assess whether INTERVAL produces larger effect sizes for change in aerobic capacity [peak $\mathrm{VO}_{2}$, oxygen consumption at anaerobic threshold $\left(\mathrm{VO}_{2}\right.$ at $\left.\mathrm{AT}\right), \mathrm{VE} / \mathrm{VCO}_{2}$ slope, respiratory exchange ratio (RER), peak minute ventilation (peak $\mathrm{V}_{\mathrm{E}}$ ), peak heart rate (PHR)], and physiological and clinical parameters compared with CONTINUOUS among patients with known cardiac disease (including CAD and CHF). The hypothesis of our study was that INTERVAL will have a greater effect on aerobic capacity given the superior improvement in mitochondrial function and cardiac contractility.

\section{Methods}

We conducted this study according to the methods of the Cochrane Handbook for Systematic Reviews of Interventions [17].

2.1. Search Strategy. The PubMed, Cochrane Library, EMBASE, and Web of Science electronic databases were searched to identify relevant clinical trials published between the earliest available date and December 2016 using the keywords "heart failure," "coronary artery disease," "high intensity interval training," "interval exercise," and "high-intensity interval exercise." The reference lists of retrieved articles were also searched to identify other appropriate studies.
2.2. Inclusion and Exclusion Criteria. Only full-text Englishlanguage reports of clinical trials were considered for inclusion. In addition, we considered only studies that compared outcomes between an intervention group performing INTERVAL and a control group performing CONTINUOUS, with rhythmic aerobic exercise programs lasting at least 4 weeks. Eligible studies also reported on at least one cardiorespiratory exercise training outcome measure in patients with cardiac disease. Reviews, cases reports, editorial comment, communications, and reports without sufficient data were excluded in our meta-analysis.

2.3. Study Selection. Figure 1 illustrates the flow of study selection. Two reviewers independently screened article titles and abstracts, excluding irrelevant studies. Full texts were then reviewed, and any study not fulfilling the inclusion criteria was excluded. Differences in the assessment of study eligibility were resolved by discussion.

2.4. Data Extraction and Management. One reviewer collected the data and the second reviewer rechecked it. Collected data included authors' names, year of publication, country in which the study was conducted, duration of the trial period, participant characteristics, intervention description, and outcomes assessed [peak $\mathrm{VO}_{2}, \mathrm{VE} / \mathrm{VCO}_{2}$ slope, RER, peak $\mathrm{V}_{\mathrm{E}}, \mathrm{PHR}, \mathrm{VO}_{2}$ at $\mathrm{AT}$, body mass, blood pressure, blood lipid parameters, FMD findings, and left ventricular ejection fraction (LVEF)]. Disagreements regarding the data collected were resolved by consensus.

2.5. Quality Assessment. The Cochrane collaboration's tool for assessing risk of bias was used for assessing the quality of randomized controlled trials (RCTs) and Physiotherapy Evidence Database (PEDro) scale nonrandomized controlled studies, respectively $[17,18]$.

2.6. Statistical Analysis. The Cochrane Collaboration software (RevMan 5.3; Cochrane Collaboration, Oxford, UK) was used for meta-analyses. We calculated effect sizes by subtracting preintervention from postintervention values. When only baseline and postintervention standard deviations (SDs) were reported, the following formula was used to obtain the missing change value $[17]: \mathrm{SD}_{\text {change }}=\sqrt{ }\left[\left(\mathrm{SD}_{\text {pre }}\right)^{2}\right.$ $+\left(\mathrm{SD}_{\text {post }}\right)^{2}-2 \times \operatorname{corr}($ pre, post $\left.) \times \mathrm{SD}_{\text {pre }} \times \mathrm{SD}_{\text {post }}\right]$, where corr is the correlation coefficient calculated for each outcome using the formula of Conraads et al. [10]: corr $=\left(\mathrm{SD}_{\text {pre }}{ }^{2}+\right.$ $\left.\mathrm{SD}_{\text {post }}{ }^{2}-\mathrm{SD}_{\text {change }}{ }^{2}\right) /\left(2 \times \mathrm{SD}_{\text {pre }} \times \mathrm{SD}_{\text {post }}\right)$. The heterogeneity of included trials was assessed using the $I^{2}$ statistic and the chi-squared test for heterogeneity. We used a fixed-effects model for studies showing significant homogeneity $\left(I^{2}<\right.$ $50 \%)$ and a random-effects model for other studies. Results were considered significant when $p<0.05$. To determine the influence of individual studies on the results obtained, we conducted a sensitivity analysis with one-by-one removal of studies. Publication bias was investigated using funnel plots and Egger's regression model. 


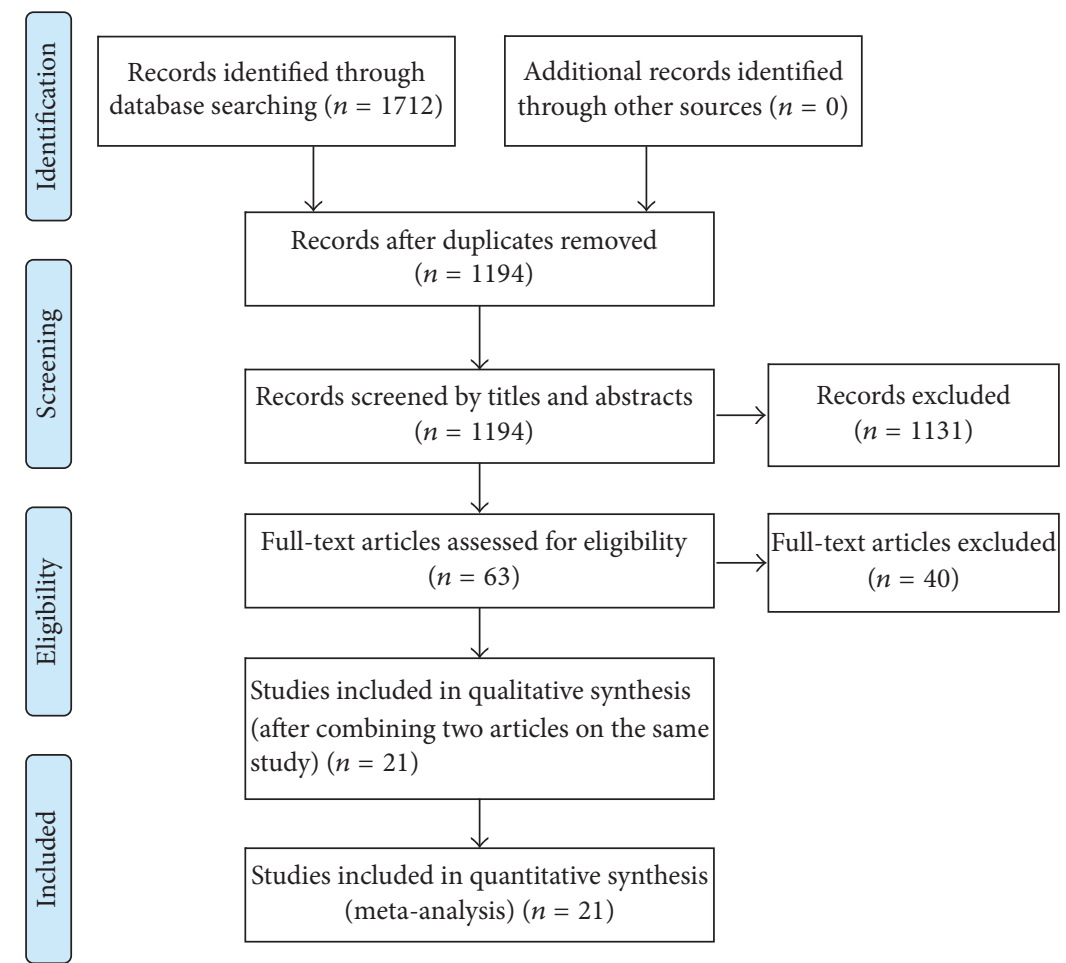

FIgURE 1: Flow chart of the study selection procedure.

\section{Results}

3.1. Characteristics of Identified Studies. The database search yielded 1712 titles. After the removal of duplicate records and the screening of abstracts and titles to assess relevance, 63 studies were selected for full-text review. After the exclusion of 40 articles which did not comply with the inclusion criteria, the final sample consisted of 23 articles $[9,10,12$, 13, 19-37] that reported on 21 studies. The characteristics of included studies are summarized in Table 1. All included studies were the randomized controlled trials. The 21 studies involved a total of 736 patients ( $81 \%$ male, $19 \%$ female) with cardiac disease (eleven studies examined patients with CAD and ten studies examined those with CHF). Four studies were conducted in Norway, three were conducted in Brazil, two each were conducted in the United States, Greece, and Canada, and one each was conducted in the Republic of Korea, Belgium, Netherlands, France, Taiwan, Italy, Spain, and United Kingdom. The duration of training programs ranged from 4 to 24 weeks, and the frequency of exercise training ranged from 2 to 5 days/week.

3.2. Risk of Bias. Figure 2 shows the risk of bias of the selected studies. Six (28.5\%) studies described the methods used to generate and conceal allocation sequences. Participants were not blinded in any study. Outcome assessors were blinded to treatment allocation in sixteen (76.2\%) studies. Seventeen $(80.9 \%)$ studies had incomplete descriptions of outcomes, and eleven $(52.3 \%)$ studies had low risks of selective reporting bias.

\subsection{Effects of Interventions on the Cardiorespiratory Measurements}

3.3.1. Peak $\mathrm{VO}_{2}$. The authors of 21 studies [9, 10, 12, 13, 19-37] involving 738 patients reported on peak oxygen uptake following INTERVAL and CONTINUOUS. Peak $\mathrm{VO}_{2}$ improved by $1.76 \mathrm{~mL} / \mathrm{kg} / \mathrm{min}$ [95\% confidence interval (CI) 1.06 to $2.46 \mathrm{~mL} / \mathrm{kg} / \mathrm{min}$ ] among patients in the INTERVAL groups, which was greater than observed in the CONTINUOUS groups, based on a random-effects model (overall $Z=4.92$, $p<0.001$ ). However, this outcome showed significant heterogeneity $\left(I^{2}=60 \%, p<0.001\right.$; Figure 3$)$.

There was significant heterogeneity in the study outcomes. Therefore, subgroup analysis was performed based on the patient's mean age and disease types. INTERVAL led to significantly greater improvements in peak $\mathrm{VO}_{2}$ than did CONTINUOUS in patients aged $<60$ years [mean difference (MD) $1.80 \mathrm{~mL} / \mathrm{kg} / \mathrm{min}, 95 \%$ CI 1.10 to $2.50 \mathrm{~mL} / \mathrm{kg} / \mathrm{min}, p<$ $0.001, I^{2}=22 \%$ ], those aged $61-75$ years $(\mathrm{MD} 1.10 \mathrm{~mL} / \mathrm{kg} / \mathrm{min}$, $95 \%$ CI 0.36 to $1.83 \mathrm{~mL} / \mathrm{kg} / \mathrm{min}, p=0.003, I^{2}=0 \%$ ), and those aged $>75$ years (included in only one study [12]; Figure 4). From disease types subgroup analyses, INTERVAL also led to significantly greater improvements in peak $\mathrm{VO}_{2}$ than did CONTINUOUS in patients with CAD (MD $1.62 \mathrm{~mL} / \mathrm{kg} / \mathrm{min}$, 95\% CI 0.94 to $\left.2.30 \mathrm{~mL} / \mathrm{kg} / \mathrm{min}, p<0.001, I^{2}=14 \%\right)$ and those with CHF (MD $1.70 \mathrm{~mL} / \mathrm{kg} / \mathrm{min}, 95 \%$ CI 0.53 to $2.86 \mathrm{~mL} / \mathrm{kg} / \mathrm{min}, p=0.004, I^{2}=73 \%$; Figure 5).

Sensitivity analysis did not change the statistical significance of the overall results. Exclusion of the study conducted 


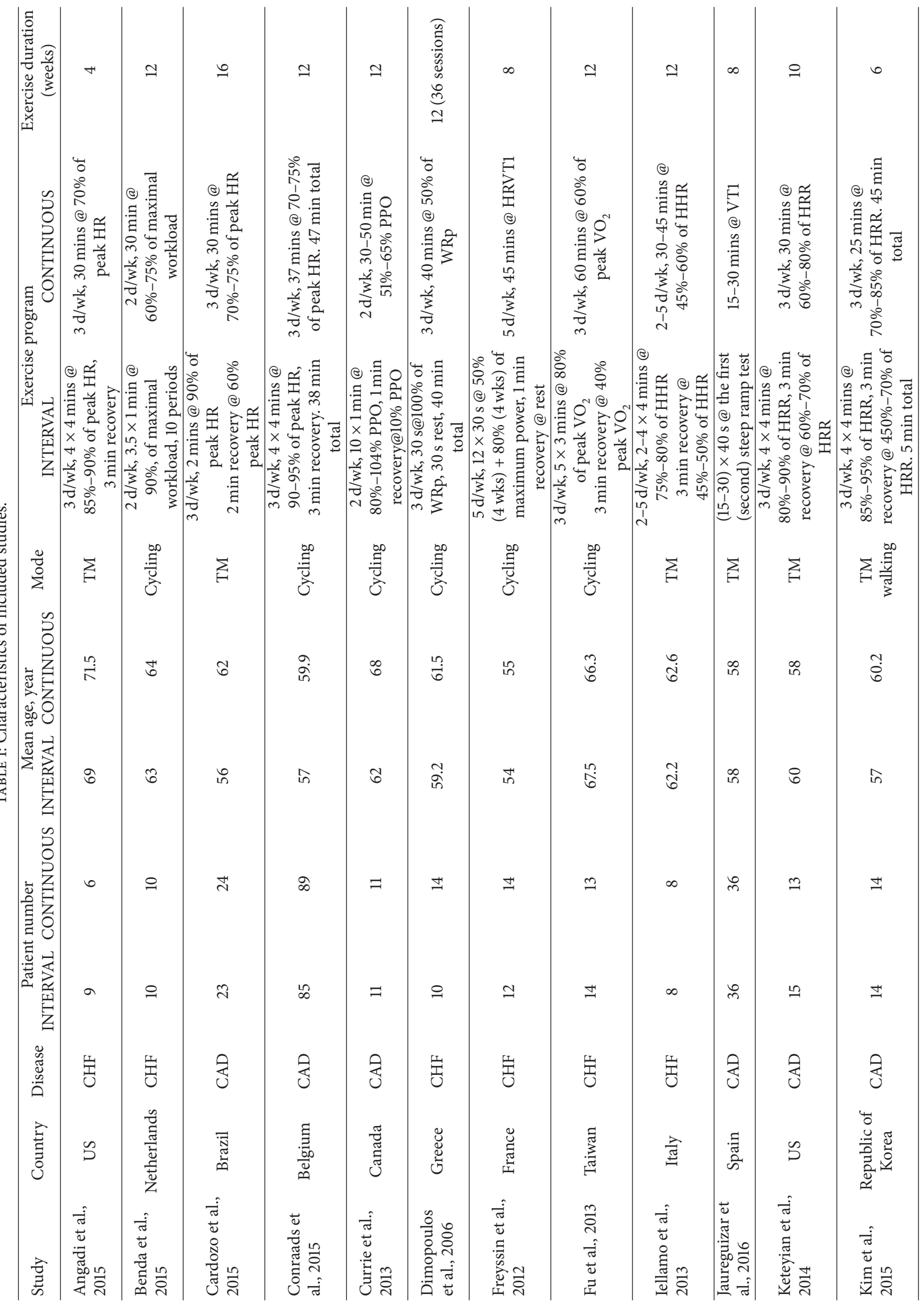




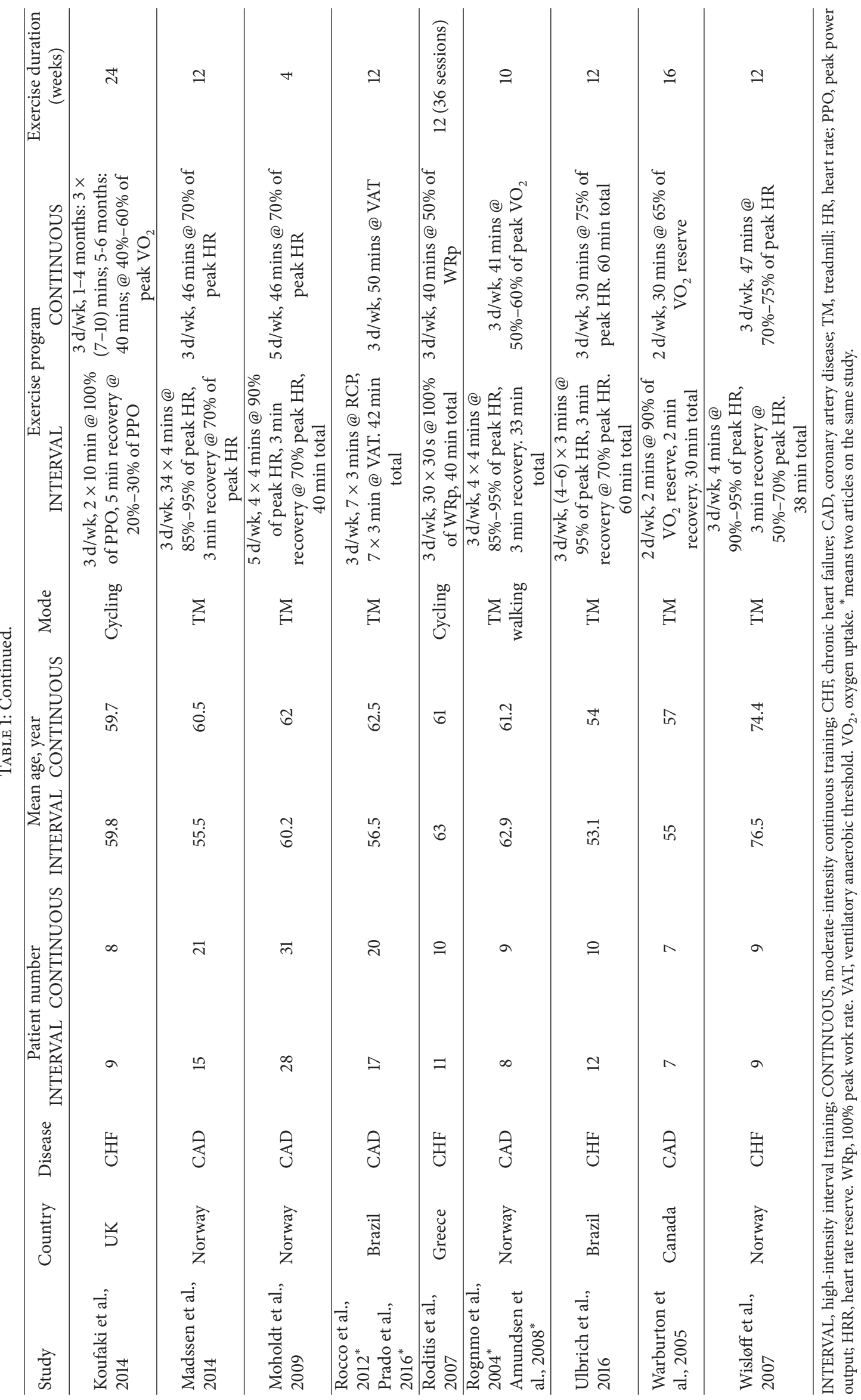




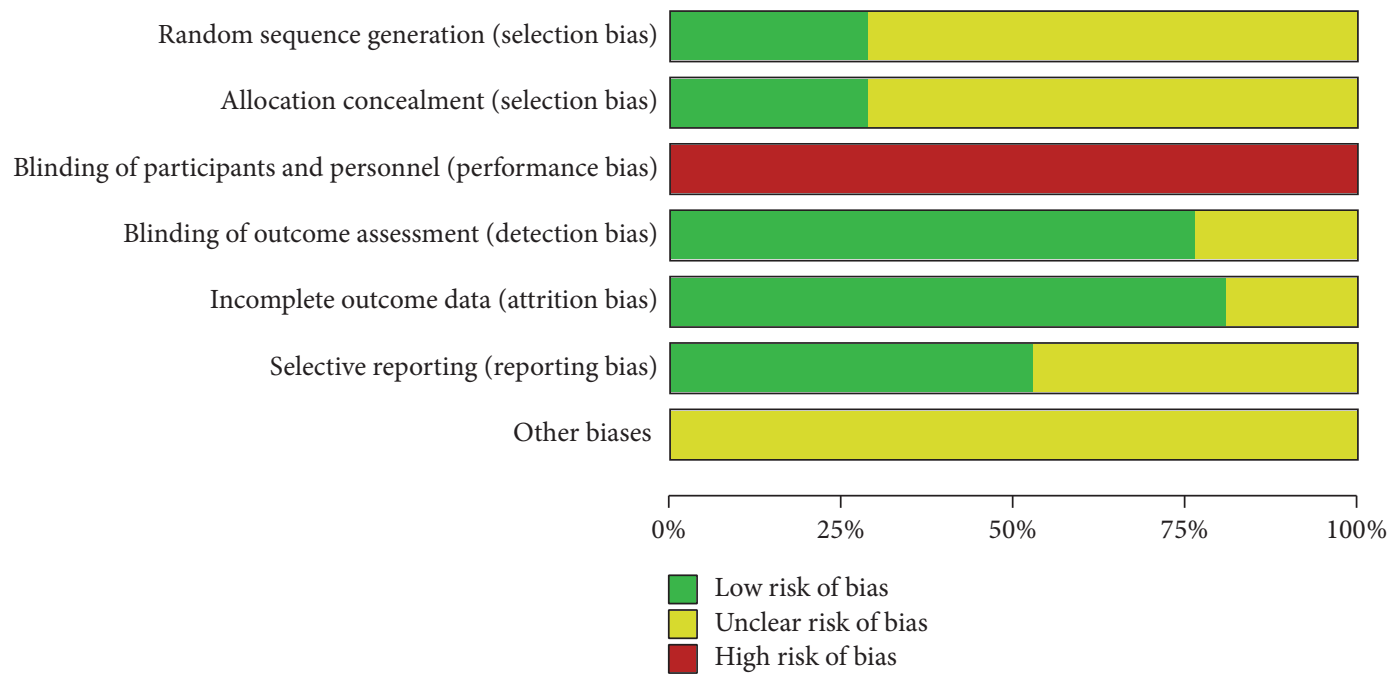

FIGURE 2: Quality assessment of RCTs using Cochrane collaboration's tool for assessing risk of bias.

\begin{tabular}{|c|c|c|c|c|c|c|c|c|c|c|c|}
\hline \multirow{3}{*}{$\begin{array}{l}\text { Study or subgroup } \\
\text { Angadi et al. } 2015\end{array}$} & \multicolumn{3}{|c|}{ INTERVAL } & \multicolumn{3}{|c|}{ CONTINUOUS } & \multirow{2}{*}{ Weight } & \multirow{2}{*}{$\begin{array}{c}\text { Mean difference } \\
\text { IV, random, 95\% CI }\end{array}$} & \multirow{2}{*}{\multicolumn{3}{|c|}{$\begin{array}{c}\text { Mean difference } \\
\text { IV, random, } 95 \% \mathrm{CI}\end{array}$}} \\
\hline & Mean & SD & Total & Mean & $\mathrm{SD}$ & Total & & & & & \\
\hline & 1.8 & 2.94 & 9 & -0.1 & 2.2 & 6 & $4.0 \%$ & $1.90[-0.71,4.51]$ & & & \\
\hline Benda et al. 2015 & 1.3 & 2.38 & 10 & 0.1 & 2.07 & 10 & $5.3 \%$ & $1.20[-0.75,3.15]$ & & & \\
\hline Cardozo et al. 2015 & 3.8 & 2.83 & 23 & 0.1 & 3.39 & 24 & $5.7 \%$ & $3.70[1.92,5.48]$ & & & \\
\hline Conraads et al. 2015 & 5.1 & 3.75 & 85 & 4.4 & 3.64 & 89 & $7.5 \%$ & $0.70[-0.40,1.80]$ & & & \\
\hline Currie et al. 2013 & 4.7 & 2.44 & 11 & 3.6 & 3.36 & 10 & $4.2 \%$ & $1.10[-1.43,3.63]$ & & & \\
\hline Dimopoulos et al. 2006 & 1.2 & 2.72 & 10 & 0.9 & 2.12 & 14 & $5.2 \%$ & $0.30[-1.72,2.32]$ & & & \\
\hline Freyssin et al. 2012 & 2.9 & 1.75 & 12 & 0.2 & 2.32 & 14 & $6.3 \%$ & $2.70[1.13,4.27]$ & & & \\
\hline Fu et al. 2013 & 3.6 & 2.44 & 14 & 0.1 & 3.56 & 13 & $4.6 \%$ & $3.50[1.18,5.82]$ & & & \\
\hline Iellamo et al. 2013 & 4.24 & 2.52 & 8 & 4.09 & 2.38 & 8 & $4.4 \%$ & $0.15[-2.25,2.55]$ & & & \\
\hline Jaureguizar et al. 2016 & 4.5 & 4.7 & 36 & 2.5 & 3.6 & 36 & $5.4 \%$ & $2.00[0.07,3.93]$ & & & \\
\hline Keteyian et al. 2014 & 3.6 & 3.1 & 15 & 1.7 & 1.7 & 13 & $5.6 \%$ & $1.90[0.08,3.72]$ & & & \\
\hline Kim et al. 2015 & 6.46 & 4.31 & 14 & 2.47 & 4.78 & 14 & $2.9 \%$ & $3.99[0.62,7.36]$ & & & \\
\hline Koufaki et al. 2014 & 2.4 & 2.72 & 8 & 1.3 & 4.15 & 9 & $3.0 \%$ & $1.10[-2.20,4.40]$ & & & \\
\hline Madssen et al. 2014 & 3.3 & 5.5 & 15 & 2 & 7.77 & 21 & $2.0 \%$ & $1.30[-3.03,5.63]$ & & & \\
\hline Moholdt et al. 2009 & 3.3 & 2.99 & 28 & 2.3 & 3.08 & 31 & $6.3 \%$ & $1.00[-0.55,2.55]$ & & & \\
\hline Rocco et al. 2012 & 4.4 & 2.48 & 17 & 3.4 & 3.19 & 20 & $5.6 \%$ & $1.00[-0.83,2.83]$ & & & \\
\hline Roditis et al. 2007 & 1.2 & 2.32 & 11 & 1.3 & 2.52 & 10 & $5.1 \%$ & $-0.10[-2.18,1.98]$ & & & \\
\hline Rognmo et al. 2004 & 6 & 6.82 & 8 & 2.7 & 3.13 & 9 & $1.5 \%$ & $3.30[-1.85,8.45]$ & & & \\
\hline Ulbrich et al. 2016 & 2.79 & 2.51 & 12 & 1.84 & 2.41 & 10 & $5.1 \%$ & $0.95[-1.11,3.01]$ & & & \\
\hline Warburton et al. 2005 & 5.16 & 5.01 & 7 & 4.01 & 2.69 & 7 & $2.1 \%$ & $1.15[-3.06,5.36]$ & & & \\
\hline Wisløff et al. 2007 & 6 & 1.15 & 9 & 1.9 & 0.6 & 8 & $8.0 \%$ & $4.10[3.24,4.96]$ & & & - \\
\hline Total (95\% CI) & & & 362 & & & 376 & $100.0 \%$ & $1.76[1.06,2.46]$ & & & \\
\hline \multirow{2}{*}{\multicolumn{9}{|c|}{$\begin{array}{l}\text { Heterogeneity: } \tau^{2}=1.41 ; \chi^{2}=50.09, \mathrm{df}=20(p=0.0002) ; I^{2}=60 \% \\
\text { Test for overall effect: } Z=4.92(p<0.00001)\end{array}$}} & -4 & 0 & 4 \\
\hline & & & & & & & & & Favours & $\mathrm{Fa}$ & avours \\
\hline
\end{tabular}

FIGURE 3: Meta-analysis of effects of INTERVAL on peak $\mathrm{VO}_{2}$.

by Wisløff et al. [12], which provided inferior evidence for the effect of INTERVAL on peak $\mathrm{VO}_{2}$, significantly improved homogeneity.

3.3.2. $\mathrm{VO}_{2}$ at $A T$. The authors of fourteen studies $[12,13$, $20,22-28,30,34,35,37]$ involving 382 patients reported on $\mathrm{VO}_{2}$ at AT following INTERVAL and CONTINUOUS. $\mathrm{VO}_{2}$ at AT improved by $0.90 \mathrm{~mL} / \mathrm{kg} / \mathrm{min}$ [95\% CI 0.08 to
$1.79 \mathrm{~mL} / \mathrm{kg} / \mathrm{min}$ ] among patients in the INTERVAL groups, which was greater than observed in the CONTINUOUS groups, based on a random-effects model (overall $Z=$ 2.14, $p=0.03$ ). However, this outcome showed significant heterogeneity $\left(I^{2}=83 \%, p<0.001\right.$; Figure 6).

3.3.3. Peak Heart Rate. The authors of seventeen studies $[10,12,13,20-23,25-29,31,33,35-37]$ involving 611 patients 


\begin{tabular}{|c|c|c|c|c|c|c|c|c|c|c|c|}
\hline \multirow{2}{*}{ Study or subgroup } & \multicolumn{3}{|c|}{ INTERVAL } & \multicolumn{3}{|c|}{ CONTINUOUS } & \multirow{2}{*}{ Weight } & \multirow{2}{*}{$\begin{array}{l}\text { Mean difference } \\
\text { IV, random, 95\% CI }\end{array}$} & \multirow{2}{*}{\multicolumn{3}{|c|}{$\begin{array}{l}\text { Mean difference } \\
\text { IV, random, 95\% CI }\end{array}$}} \\
\hline & Mean & $\mathrm{SD}$ & Total & Mean & $\mathrm{SD}$ & Total & & & & & \\
\hline \multicolumn{12}{|l|}{ 1.3.1 Age $<60$ years } \\
\hline Cardozo et al. 2015 & 3.8 & 2.83 & 23 & 0.1 & 3.39 & 24 & $5.7 \%$ & $3.70[1.92,5.48]$ & & & \\
\hline Conraads et al. 2015 & 5.1 & 3.75 & 85 & 4.4 & 3.64 & 89 & $7.5 \%$ & $0.70[-0.40,1.80]$ & & & \\
\hline Freyssin et al. 2012 & 2.9 & 1.75 & 12 & 0.2 & 2.32 & 14 & $6.3 \%$ & $2.70[1.13,4.27]$ & & & \\
\hline Jaureguizar et al. 2016 & 4.5 & 4.7 & 36 & 2.5 & 3.6 & 36 & $5.4 \%$ & $2.00[0.07,3.93]$ & & & \\
\hline Keteyian et al. 2014 & 3.6 & 3.1 & 15 & 1.7 & 1.7 & 13 & $5.6 \%$ & $1.90[0.08,3.72]$ & & & \\
\hline Kim et al. 2015 & 6.46 & 4.31 & 14 & 2.47 & 4.78 & 14 & $2.9 \%$ & $3.99[0.62,7.36]$ & & & \\
\hline Koufaki et al. 2014 & 2.4 & 2.72 & 8 & 1.3 & 4.15 & 9 & $3.0 \%$ & $1.10[-2.20,4.40]$ & & & \\
\hline Madssen et al. 2014 & 3.3 & 5.5 & 15 & 2 & 7.77 & 21 & $2.0 \%$ & $1.30[-3.03,5.63]$ & & & \\
\hline Rocco et al. 2012 & 4.4 & 2.48 & 17 & 3.4 & 3.19 & 20 & $5.6 \%$ & $1.00[-0.83,2.83]$ & & & \\
\hline Ulbrich et al. 2016 & 2.79 & 2.51 & 12 & 1.84 & 2.41 & 10 & $5.1 \%$ & $0.95[-1.11,3.01]$ & & & \\
\hline Warburton et al. 2005 & 5.16 & 5.01 & 7 & 4.01 & 2.69 & 7 & $2.1 \%$ & $1.15[-3.06,5.36]$ & & & \\
\hline Subtotal (95\% CI) & & & 244 & & & 257 & $51.3 \%$ & $1.80[1.10,2.50]$ & & & \\
\hline \multicolumn{12}{|c|}{ Heterogeneity: $\tau^{2}=0.29 ; \chi^{2}=12.78, \mathrm{df}=10(p=0.24) ; I^{2}=22 \%$} \\
\hline \multicolumn{12}{|c|}{ Test for overall effect: $Z=5.06(p<0.00001)$} \\
\hline \multicolumn{12}{|l|}{ 1.3.2 Age 60-75 years } \\
\hline Angadi et al. 2015 & 1.8 & 2.94 & 9 & -0.1 & 2.2 & 6 & $4.0 \%$ & $1.90[-0.71,4.51]$ & & & \\
\hline Benda et al. 2015 & 1.3 & 2.38 & 10 & 0.1 & 2.07 & 10 & $5.3 \%$ & $1.20[-0.75,3.15]$ & & & \\
\hline Currie et al. 2013 & 4.7 & 2.44 & 11 & 3.6 & 3.36 & 10 & $4.2 \%$ & $1.10[-1.43,3.63]$ & & & \\
\hline Dimopoulos et al. 2006 & 1.2 & 2.72 & 10 & 0.9 & 2.12 & 14 & $5.2 \%$ & $0.30[-1.72,2.32]$ & & & \\
\hline Fu et al. 2013 & 3.6 & 2.44 & 14 & 0.1 & 3.56 & 13 & $4.6 \%$ & $3.50[1.18,5.82]$ & & & \\
\hline Iellamo et al. 2013 & 4.24 & 2.52 & 8 & 4.09 & 2.38 & 8 & $4.4 \%$ & $0.15[-2.25,2.55]$ & & & \\
\hline Moholdt et al. 2009 & 3.3 & 2.99 & 28 & 2.3 & 3.08 & 31 & $6.3 \%$ & $1.00[-0.55,2.55]$ & & & \\
\hline Roditis et al. 2007 & 1.2 & 2.32 & 11 & 1.3 & 2.52 & 10 & $5.1 \%$ & $-0.10[-2.18,1.98]$ & & & \\
\hline Rognmo et al. 2004 & 6 & 6.82 & 8 & 2.7 & 3.13 & 9 & $1.5 \%$ & $3.30[-1.85,8.45]$ & & & \\
\hline Subtotal (95\% CI) & & & 109 & & & 111 & $40.6 \%$ & $1.10[0.36,1.83]$ & & & \\
\hline \multicolumn{12}{|c|}{ Heterogeneity: $\tau^{2}=0.00 ; \chi^{2}=7.69, \mathrm{df}=8(p=0.46) ; I^{2}=0 \%$} \\
\hline \multicolumn{12}{|c|}{ Test for overall effect: $Z=2.93(p=0.003)$} \\
\hline \multicolumn{12}{|l|}{ 1.3.3 Age $>75$ years } \\
\hline Wisløff et al. 2007 & 6 & 1.15 & 9 & 1.9 & 0.6 & 8 & $8.0 \%$ & $4.10[3.24,4.96]$ & & & \\
\hline Subtotal (95\% CI) & & & 9 & & & 8 & $8.0 \%$ & $4.10[3.24,4.96]$ & & & \\
\hline \multicolumn{12}{|c|}{ Heterogeneity: not applicable } \\
\hline \multicolumn{12}{|c|}{ Test for overall effect: $Z=9.36(p<0.00001)$} \\
\hline Total $(95 \%$ CI) & & & 362 & & & 376 & $100.0 \%$ & $1.76[1.06,2.46]$ & & & \\
\hline \multicolumn{9}{|c|}{$\begin{array}{l}\text { Heterogeneity: } \tau^{2}=1.41 ; \chi^{2}=50.09, \mathrm{df}=20(p=0.0002) ; I^{2}=60 \% \\
\text { Test for overall effect: } Z=4.92(p<0.00001)\end{array}$} & $\begin{array}{cc} & \\
-4 & -2\end{array}$ & 2 & 4 \\
\hline \multicolumn{12}{|c|}{$\begin{array}{l}\text { Test for subgroup differences: } \chi^{2}=28.68, \mathrm{df}=2(p<0.00001), I^{2}=93.0 \% \\
\text { Ter }\end{array}$} \\
\hline
\end{tabular}

FIGURE 4: Meta-analysis of the effects of INTERVAL on peak $\mathrm{VO}_{2}$ according to age.

reported on peak heart rate following INTERVAL and CONTINUOUS. A random-effects model revealed no significant difference between groups (MD $0.97 \mathrm{bpm}, 95 \% \mathrm{CI}-2.19$ to $4.12 \mathrm{bpm}, p=0.55)$.

3.3.4. Peak Minute Ventilation. The authors of five studies [23, 25, 35-37] involving 103 patients reported on peak VE following INTERVAL and CONTINUOUS. A randomeffects model revealed no significant difference between groups (MD 3.46 l/min, 95\% CI -1.75 to $8.671 / \mathrm{min}, p=0.19$ ).

3.3.5. VE $/ \mathrm{VCO}_{2}$ Slope. The authors of nine studies $[20-23,26$, $28,34,35,37]$ involving 220 patients reported on $\mathrm{VE} / \mathrm{VCO}_{2}$ slope following INTERVAL and CONTINUOUS. A fixedeffects model revealed no significant difference between groups (MD 0.24, $95 \% \mathrm{CI}-0.40$ to $0.87, p=0.46$ ).

3.3.6. Respiratory Exchange Ratio. The authors of fourteen studies $[10,12,13,20,22,26-33,36]$ involving 579 patients reported on RER following INTERVAL and CONTINUOUS. A random-effects model revealed no significant difference between groups (MD 0.01, 95\% CI -0.01 to $0.02, p=0.25$ ).

\subsection{Effects of Interventions on Physiological and Clinical Parameters}

3.4.1. Body Mass. The authors of eight studies [10, 13, 20, $25,32,34,36,37]$ involving 363 patients reported decreased body mass following INTERVAL and CONTINUOUS. A fixed-effects model revealed no significant difference between groups (MD $0.55 \mathrm{~kg}, 95 \% \mathrm{CI}-0.52$ to $1.62 \mathrm{~kg}, p=0.31$ ).

3.4.2. Blood Pressure. The authors of eight studies $[9,10,13$, $20,25,27,28,36]$ involving 376 patients reported on systolic blood pressure (SBP) and diastolic blood pressure (DBP) following INTERVAL and CONTINUOUS. A random-effects model revealed no significant difference between groups 


\begin{tabular}{|c|c|c|c|c|c|c|c|c|c|c|}
\hline \multirow{2}{*}{ Study or subgroup } & \multicolumn{3}{|c|}{ INTERVAL } & \multicolumn{3}{|c|}{ CONTINUOUS } & \multirow{2}{*}{ Weight } & \multirow{2}{*}{$\begin{array}{l}\text { Mean difference } \\
\text { IV, random, 95\% CI }\end{array}$} & \multirow{2}{*}{\multicolumn{2}{|c|}{$\begin{array}{l}\text { Mean difference } \\
\text { IV, random, 95\% CI }\end{array}$}} \\
\hline & Mean & $\mathrm{SD}$ & Total & Mean & $\mathrm{SD}$ & Total & & & & \\
\hline \multicolumn{11}{|l|}{$1.2 .1 C A D$} \\
\hline Cardozo et al. 2015 & 3.8 & 2.83 & 23 & 0.1 & 3.39 & 24 & $5.7 \%$ & $3.70[1.92,5.48]$ & & \\
\hline Conraads et al. 2015 & 5.1 & 3.75 & 85 & 4.4 & 3.64 & 89 & $7.5 \%$ & $0.70[-0.40,1.80]$ & & \\
\hline Currie et al. 2013 & 4.7 & 2.44 & 11 & 3.6 & 3.36 & 10 & $4.2 \%$ & $1.10[-1.43,3.63]$ & & \\
\hline Jaureguizar et al. 2016 & 4.5 & 4.7 & 36 & 2.5 & 3.6 & 36 & $5.4 \%$ & $2.00[0.07,3.93]$ & & \\
\hline Keteyian et al. 2014 & 3.6 & 3.1 & 15 & 1.7 & 1.7 & 13 & $5.6 \%$ & $1.90[0.08,3.72]$ & & \\
\hline Kim et al. 2015 & 6.46 & 4.31 & 14 & 2.47 & 4.78 & 14 & $2.9 \%$ & $3.99[0.62,7.36]$ & & \\
\hline Madssen et al. 2014 & 3.3 & 5.5 & 15 & 2 & 7.77 & 21 & $2.0 \%$ & $1.30[-3.03,5.63]$ & & \\
\hline Moholdt et al. 2009 & 3.3 & 2.99 & 28 & 2.3 & 3.08 & 31 & $6.3 \%$ & $1.00[-0.55,2.55]$ & & \\
\hline Rocco et al. 2012 & 4.4 & 2.48 & 17 & 3.4 & 3.19 & 20 & $5.6 \%$ & $1.00[-0.83,2.83]$ & & \\
\hline Rognmo et al. 2004 & 6 & 6.82 & 8 & 2.7 & 3.13 & 9 & $1.5 \%$ & $3.30[-1.85,8.45]$ & & \\
\hline Warburton et al. 2005 & 5.16 & 5.01 & 7 & 4.01 & 2.69 & 7 & $2.1 \%$ & $1.15[-3.06,5.36]$ & & \\
\hline Subtotal (95\% CI) & & & 259 & & & 274 & $49.0 \%$ & $1.62[0.94,2.30]$ & & \\
\hline \multicolumn{11}{|c|}{ Heterogeneity: $\tau^{2}=0.19 ; \chi^{2}=11.69, \mathrm{df}=10(p=0.31) ; I^{2}=14 \%$} \\
\hline \multicolumn{11}{|c|}{ Test for overall effect: $Z=4.69(p<0.00001)$} \\
\hline \multicolumn{11}{|l|}{ 1.2.2 CHF } \\
\hline Angadi et al. 2015 & 1.8 & 2.94 & 9 & -0.1 & 2.2 & 6 & $4.0 \%$ & $1.90[-0.71,4.51]$ & & \\
\hline Benda et al. 2015 & 1.3 & 2.38 & 10 & 0.1 & 2.07 & 10 & $5.3 \%$ & $1.20[-0.75,3.15]$ & & \\
\hline Dimopoulos et al. 2006 & 1.2 & 2.72 & 10 & 0.9 & 2.12 & 14 & $5.2 \%$ & $0.30[-1.72,2.32]$ & & \\
\hline Freyssin et al. 2012 & 2.9 & 1.75 & 12 & 0.2 & 2.32 & 14 & $6.3 \%$ & $2.70[1.13,4.27]$ & & \\
\hline Fu et al. 2013 & 3.6 & 2.44 & 14 & 0.1 & 3.56 & 13 & $4.6 \%$ & $3.50[1.18,5.82]$ & & \\
\hline Iellamo et al. 2013 & 4.24 & 2.52 & 8 & 4.09 & 2.38 & 8 & $4.4 \%$ & $0.15[-2.25,2.55]$ & & \\
\hline Koufaki et al. 2014 & 2.4 & 2.72 & 8 & 1.3 & 4.15 & 9 & $3.0 \%$ & $1.10[-2.20,4.40]$ & & \\
\hline Roditis et al. 2007 & 1.2 & 2.32 & 11 & 1.3 & 2.52 & 10 & $5.1 \%$ & $-0.10[-2.18,1.98]$ & & \\
\hline Ulbrich et al. 2016 & 2.79 & 2.51 & 12 & 1.84 & 2.41 & 10 & $5.1 \%$ & $0.95[-1.11,3.01]$ & & \\
\hline Wisløff et al. 2007 & 6 & 1.15 & 9 & 1.9 & 0.6 & 8 & $8.0 \%$ & $4.10[3.24,4.96]$ & & $-\square$ \\
\hline Subtotal (95\% CI) & & & 103 & & & 102 & $51.0 \%$ & $1.70[0.53,2.86]$ & & \\
\hline \multicolumn{11}{|c|}{ Heterogeneity: $\tau^{2}=2.38 ; \chi^{2}=33.13, \mathrm{df}=9(p=0.0001) ; I^{2}=73 \%$} \\
\hline \multicolumn{11}{|c|}{ Test for overall effect: $Z=2.85(p=0.004)$} \\
\hline Total (95\% CI) & & & 362 & & & 376 & $100.0 \%$ & $1.76[1.06,2.46]$ & & \\
\hline \multicolumn{9}{|c|}{$\begin{array}{l}\text { Heterogeneity: } \tau^{2}=1.41 ; \chi^{2}=50.09, \mathrm{df}=20(p=0.0002) ; I^{2}=60 \% \\
\text { Test for overall effect: } Z=4.92(p<0.00001)\end{array}$} & -4 & 4 \\
\hline \multicolumn{9}{|c|}{ Test for subgroup differences: $\chi^{2}=0.01, \mathrm{df}=1(p=0.91), I^{2}=0 \%$} & Favours [CONTINUOUS] & Favours [INTERVAL] \\
\hline
\end{tabular}

FIgURE 5: Meta-analysis of the effects of INTERVAL on peak $\mathrm{VO}_{2}$ according to disease types.

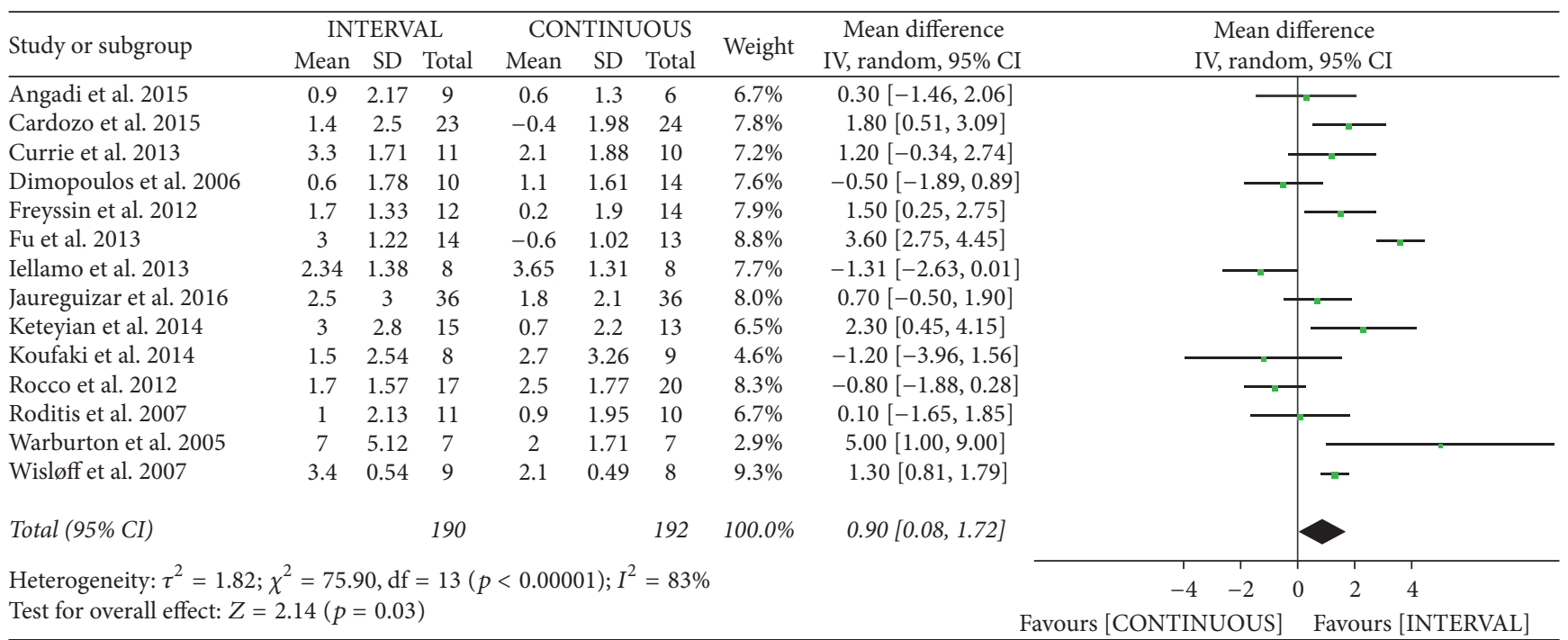




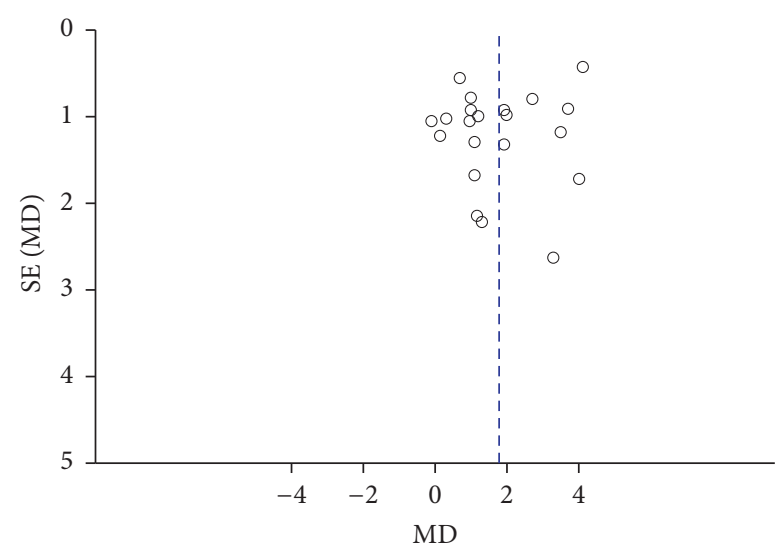

FIGURE 7: Funnel plot of publication bias.

(SBP: $\mathrm{MD}-0.09 \mathrm{mmHg}, 95 \% \mathrm{CI}-4.82$ to $4.65 \mathrm{mmHg}, p=$ 0.97; DBP: $\mathrm{MD}-0.79 \mathrm{mmHg}, 95 \% \mathrm{CI}-3.75$ to $2.16 \mathrm{mmHg}$, $p=0.60)$.

3.4.3. Blood Lipids. Data on high-density lipoprotein cholesterol (HDL-C), low-density lipoprotein cholesterol (LDLC), and triglyceride (TG) levels following INTERVAL and CONTINUOUS were reported in six studies $[22,25,26$, $29,31,32]$ involving 340 patients. A random-effects model showed no significant difference between groups (TG: standardized mean difference (SMD) $-0.05,95 \% \mathrm{CI}-0.26$ to 0.17 ; LDL-C: SMD $-0.6,95 \%$ CI -1.3 to 0.11 ; HDL-C: SMD $0.05,95 \% \mathrm{CI}-0.17$ to 0.26$)$. The result of cholesterol was assessed in four studies [22, 25, 26, 31] involving 253 patients. A random-effects model revealed no significant difference between groups (SMD 0.01, 95\% CI -0.55 to 0.57 ).

3.4.4. Flow-Mediated Dilation. The authors of six studies [10, $12,13,20,21,31]$ involving 269 patients reported on FMD following INTERVAL and CONTINUOUS. A random-effects model showed no significant difference between groups (MD $1.47 \%, 95 \% \mathrm{CI}-0.20 \%$ to $3.14 \%, p=0.09)$.

3.4.5. Left Ventricular Ejection Fraction. The authors of eight studies $[9,12,19-21,25,26,32]$ involving 170 patients reported increased LVEF following INTERVAL and CONTINUOUS. A random-effects model showed no significant difference between groups (MD 2.18\%, 95\% CI $-0.54 \%$ to $4.90 \%, p=$ $0.12)$.

3.5. Publication Bias. Egger's regression analysis excluded relevant publication bias for peak $\mathrm{VO}_{2}(p=0.14)$, and the funnel plot of these data was symmetrical.

\section{Discussion}

To our knowledge, most previous systematic reviews on this topic have focused on patients with specific diseases, such as CAD and CHF. One previous review [38] has examined whether INTERVAL is more effective than CONTINUOUS for improving peak $\mathrm{VO}_{2}$ and LVEF in CHF patients. However, this review focused only on CHF and only seven articles were included in the review. This systematic review examined the efficacy of INTERVAL as a part of cardiac rehabilitation in patients with cardiac disease (including CHF and CAD). Twenty-one studies involving 738 cardiac patients were included in the review. The main findings were that INTERVAL appears to be at least as effective as and in some cases more effective than CONTINUOUS, for the improvement of aerobic capacity, although we found evidence of heterogeneity among studies. Heterogeneous results for this outcome in the study conducted by Wisløff et al. [12] were due mainly to the inclusion of elderly patients.

4.1. Rationale and Potential Working Mechanisms of INTER$V A L$. Due to repeated alternation of high- and low-intensity exercise, INTERVAL's stimulation of the body fluctuates. The rationale is to accumulate more time in high-intensity zones compared to a continuous exercise where exhaustion would occur more prematurely and therefore to produce a stronger stimulus for cardiovascular and muscular adaptations [39, 40]. The mechanisms involved in the superiority of INTERVAL to CONTINUOUS have not been clearly elucidated. The potential mechanisms for the greater improvement in aerobic capacity achieved by INTERVAL include increased activation of peroxisome-proliferator activated receptor $\gamma$ coactivator (PGC-1 $\alpha$ ), which improves mitochondrial function [12, 41, 42], and increased maximal rate of $\mathrm{Ca}^{2+}$ reuptake into the sarcoplasmic reticulum, which reduces skeletal muscle fatigue $[12,42]$. The increase in PGC- $1 \alpha$ to be strongly correlated with the improved $\mathrm{VO}_{2}$ peak $(r=0.72, p<0.01)$ was found by Wisløff et al. [12], supporting the influence of mitochondrial function on exercise capacity.

INTERVAL has been demonstrated to activate p38 mitogen-activated protein kinase and $5^{\prime}$-adenosine monophosphate-activated protein kinase. Both of these exerciseresponsive signaling kinases are implicated in direct phosphorylation and activation of PGC- $1 \alpha$. Increased nuclear abundance of PGC- $1 \alpha$ following INTERVAL may coactivate transcription factors to increase mitochondrial gene transcription, ultimately resulting in accumulation of more mitochondrial proteins to drive mitochondrial biogenesis [43]. Mitochondrial biogenesis is essential to maintain the structural integrity of skeletal muscle. Mitochondrial function is associated with aerobic physical fitness and plays an important pathophysiological role in cardiac patients. Consequently, the major benefits of INTERVAL interventions include enhanced peripheral blood circulation [44], as well as increased skeletal muscle and functional capacity [45-47]. The improvement of peak $\mathrm{VO}_{2}$, a strong, independent predictor of all-cause and cardiovascular-specific mortality $[48,49]$, through INTERVAL is thus of clinical significance.

The magnitude of difference in the effects of INTERVAL and CONTINUOUS in terms of $\mathrm{VE} / \mathrm{VCO}_{2}$ slope, RER, peak $\mathrm{V}_{\mathrm{E}}$, PHR, body mass, blood pressure, blood lipids, FMD, and LVEF was small in the present analysis, which may be related to the examination of short-term outcomes in the included studies. Thus, more research is necessary to provide information on the long-term effects of INTERVAL. 


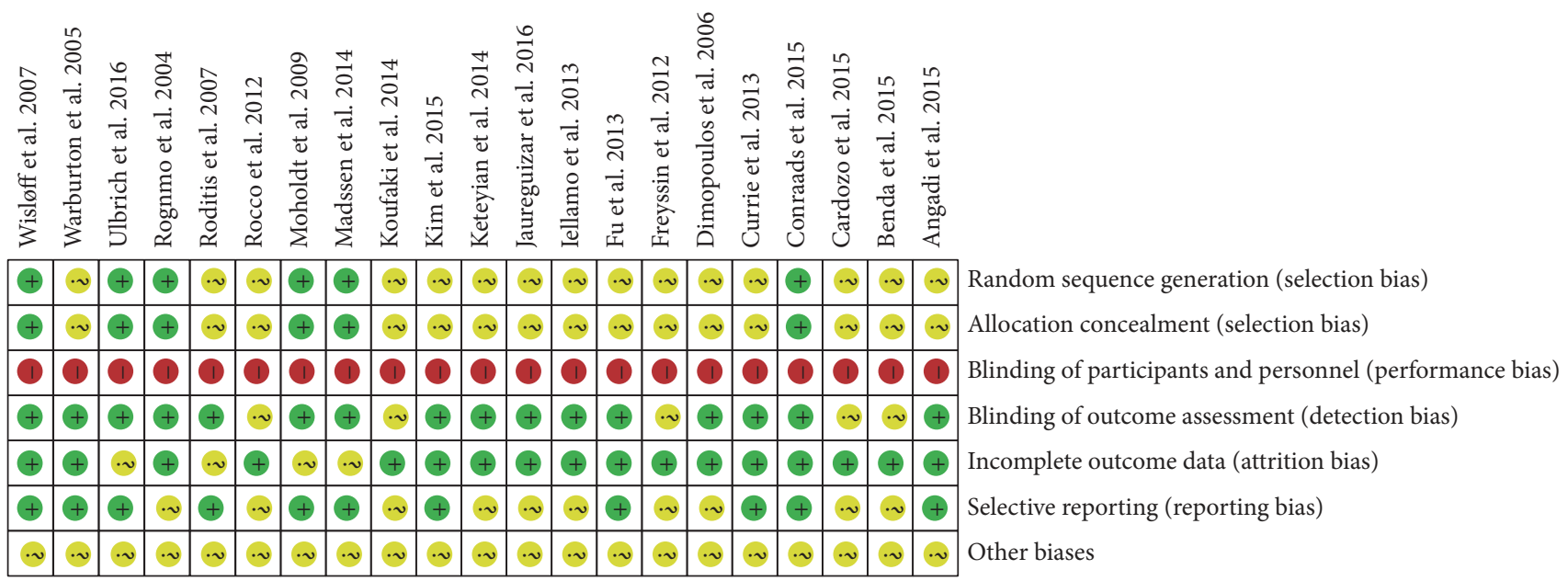

FIGURE 8: Risk of bias summary.

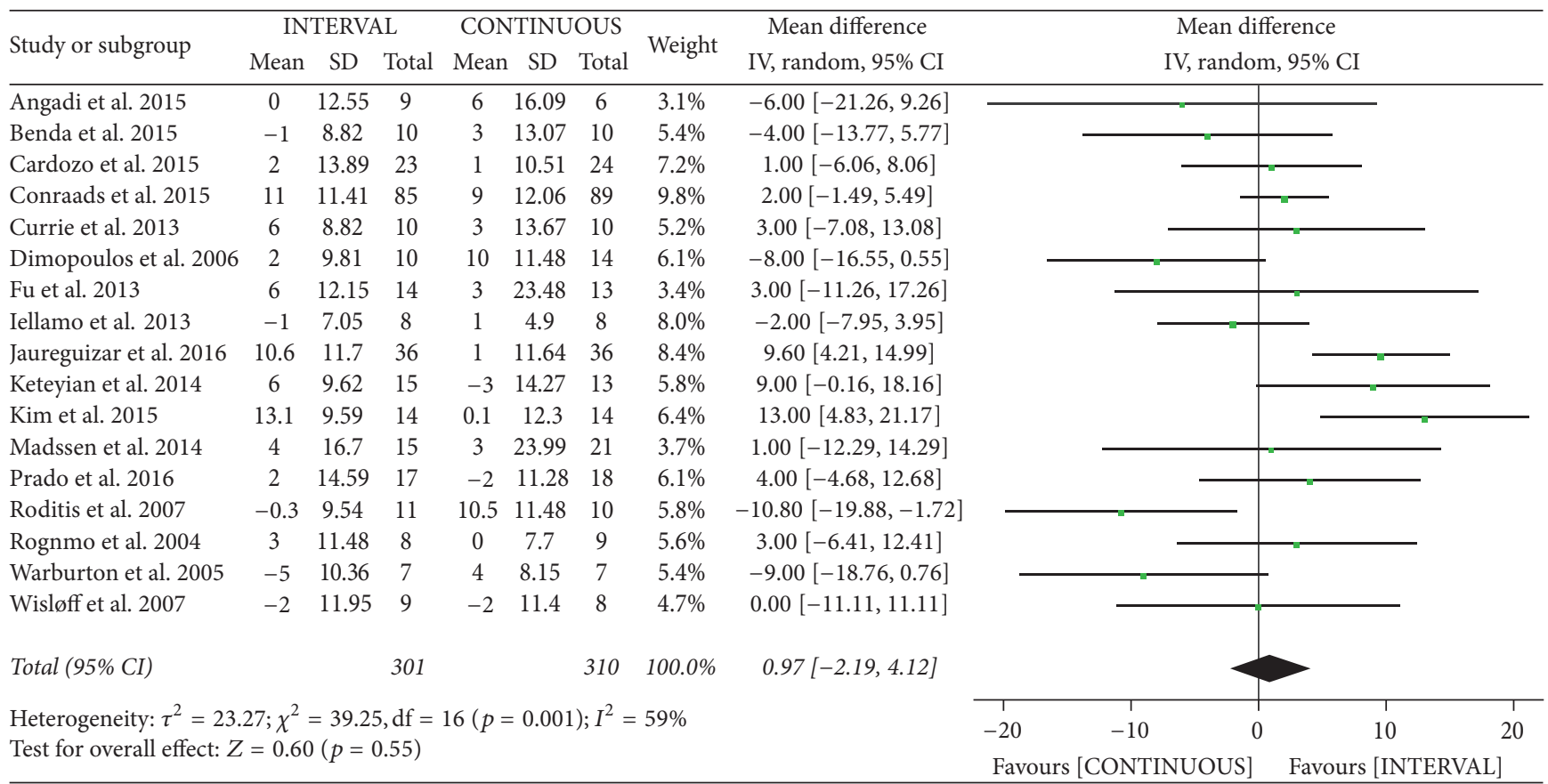

FIgURE 9: Meta-analysis of effects of INTERVAL on PHR.

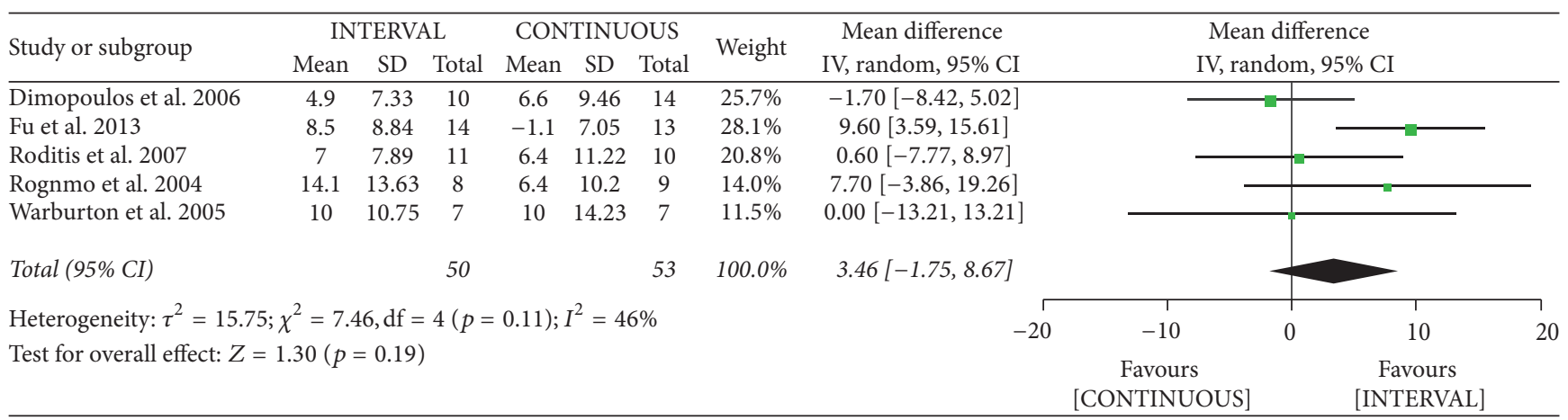

FIGURE 10: Meta-analysis of effects of INTERVAL on peak VE. 


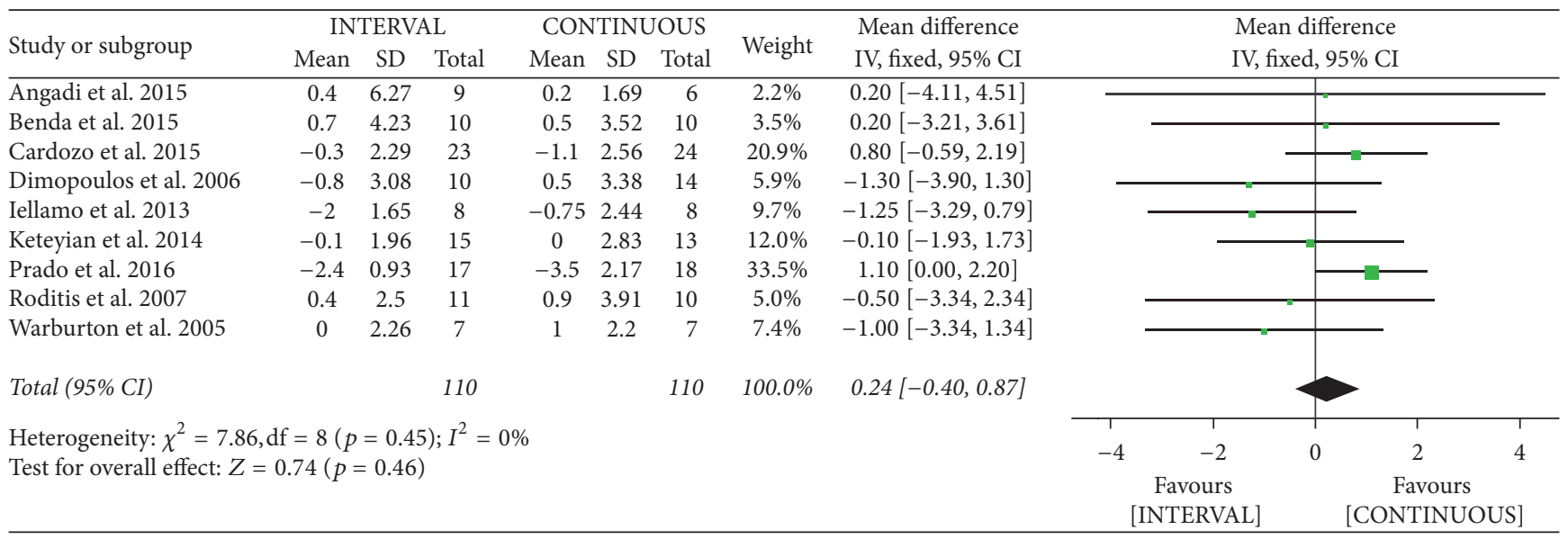

FIGURe 11: Meta-analysis of effects of INTERVAL on VE/VCO 2 slope.

\begin{tabular}{|c|c|c|c|c|c|c|c|c|c|c|c|c|}
\hline \multirow{2}{*}{ Study or subgroup } & \multicolumn{3}{|c|}{ INTERVAL } & \multicolumn{3}{|c|}{ CONTINUOUS } & \multirow{2}{*}{ Weight } & \multirow{2}{*}{$\begin{array}{c}\text { Mean difference } \\
\text { IV, random, 95\% CI }\end{array}$} & \multirow{2}{*}{\multicolumn{4}{|c|}{$\begin{array}{c}\text { Mean difference } \\
\text { IV, random, 95\% CI }\end{array}$}} \\
\hline & Mean & $\mathrm{SD}$ & Total & Mean & $\mathrm{SD}$ & Total & & & & & & \\
\hline Angadi et al. 2015 & 0.07 & 0.07 & 9 & -0.01 & 0.05 & 6 & $4.6 \%$ & $0.08[0.02,0.14]$ & & & & \\
\hline Cardozo et al. 2015 & 0.02 & 0.06 & 23 & -0.01 & 0.06 & 24 & $8.6 \%$ & $0.03[-0.00,0.06]$ & & & & \\
\hline Conraads et al. 2015 & 0.02 & 0.07 & 85 & 0.01 & 0.06 & 89 & $12.0 \%$ & $0.01[-0.01,0.03]$ & & & & \\
\hline Currie et al. 2013 & -0.02 & 0.07 & 9 & 0.04 & 0.04 & 9 & $5.5 \%$ & $-0.06[-0.11,-0.01]$ & & & & \\
\hline Iellamo et al. 2013 & -0.01 & 0.04 & 8 & 0.02 & 0.04 & 8 & $7.6 \%$ & $-0.03[-0.07,0.01]$ & & & & \\
\hline Jaureguizar et al. 2016 & 0.04 & 0.1 & 36 & 0.03 & 0.1 & 36 & $6.4 \%$ & $0.01[-0.04,0.06]$ & & & & \\
\hline Keteyian et al. 2014 & 0.03 & 0.06 & 15 & -0.01 & 0.05 & 13 & $7.4 \%$ & $0.04[-0.00,0.08]$ & & & & \\
\hline Kim et al. 2015 & 0.01 & 0.08 & 14 & 0.08 & 0.09 & 14 & $4.3 \%$ & $-0.07[-0.13,-0.01]$ & & & & \\
\hline Koufaki et al. 2014 & 0 & 0.06 & 8 & -0.02 & 0.08 & 9 & $4.0 \%$ & $0.02[-0.05,0.09]$ & & & & \\
\hline Madssen et al. 2014 & 0 & 0.05 & 15 & 0 & 0.06 & 21 & $8.3 \%$ & $0.00[-0.04,0.04]$ & & & & \\
\hline Moholdt et al. 2009 & 0.01 & 0.04 & 28 & 0 & 0.04 & 31 & $11.8 \%$ & $0.01[-0.01,0.03]$ & & & & \\
\hline Prado et al. 2016 & -0.04 & 0.46 & 17 & -0.02 & 0.48 & 18 & $0.2 \%$ & $-0.02[-0.33,0.29]$ & $\leftarrow$ & & & \\
\hline Rognmo et al. 2004 & 0.03 & 0.04 & 8 & 0.02 & 0.02 & 9 & $9.4 \%$ & $0.01[-0.02,0.04]$ & & & & \\
\hline Wisløff et al. 2007 & 0.03 & 0.03 & 9 & -0.01 & 0.03 & 8 & $9.9 \%$ & $0.04[0.01,0.07]$ & & & & \\
\hline Total (95\% CI) & & & 284 & & & 295 & $100.0 \%$ & $0.01[-0.01,0.02]$ & & & & \\
\hline \multirow{2}{*}{\multicolumn{9}{|c|}{$\begin{array}{l}\text { Heterogeneity: } \tau^{2}=0.00 ; \chi^{2}=30.04, \mathrm{df}=13(p=0.005) ; I^{2}=57 \% \\
\text { Test for overall effect: } Z=1.15(p=0.25)\end{array}$}} & -0.1 & -0.05 & 0.05 & 0.1 \\
\hline & & & & & & & & & Favours [CONT & INUOUS] & Favours & s [INTERVAL] \\
\hline
\end{tabular}

FIGURE 12: Meta-analysis of effects of INTERVAL on RER.

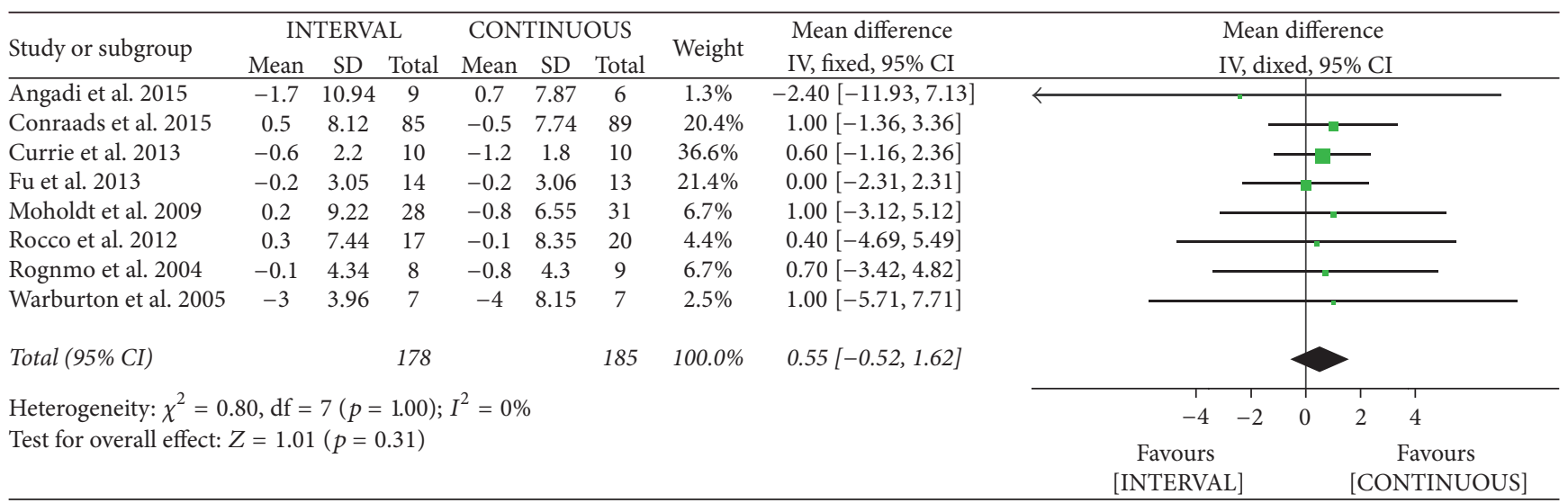

FIGURE 13: Meta-analysis of effects of INTERVAL on body mass. 


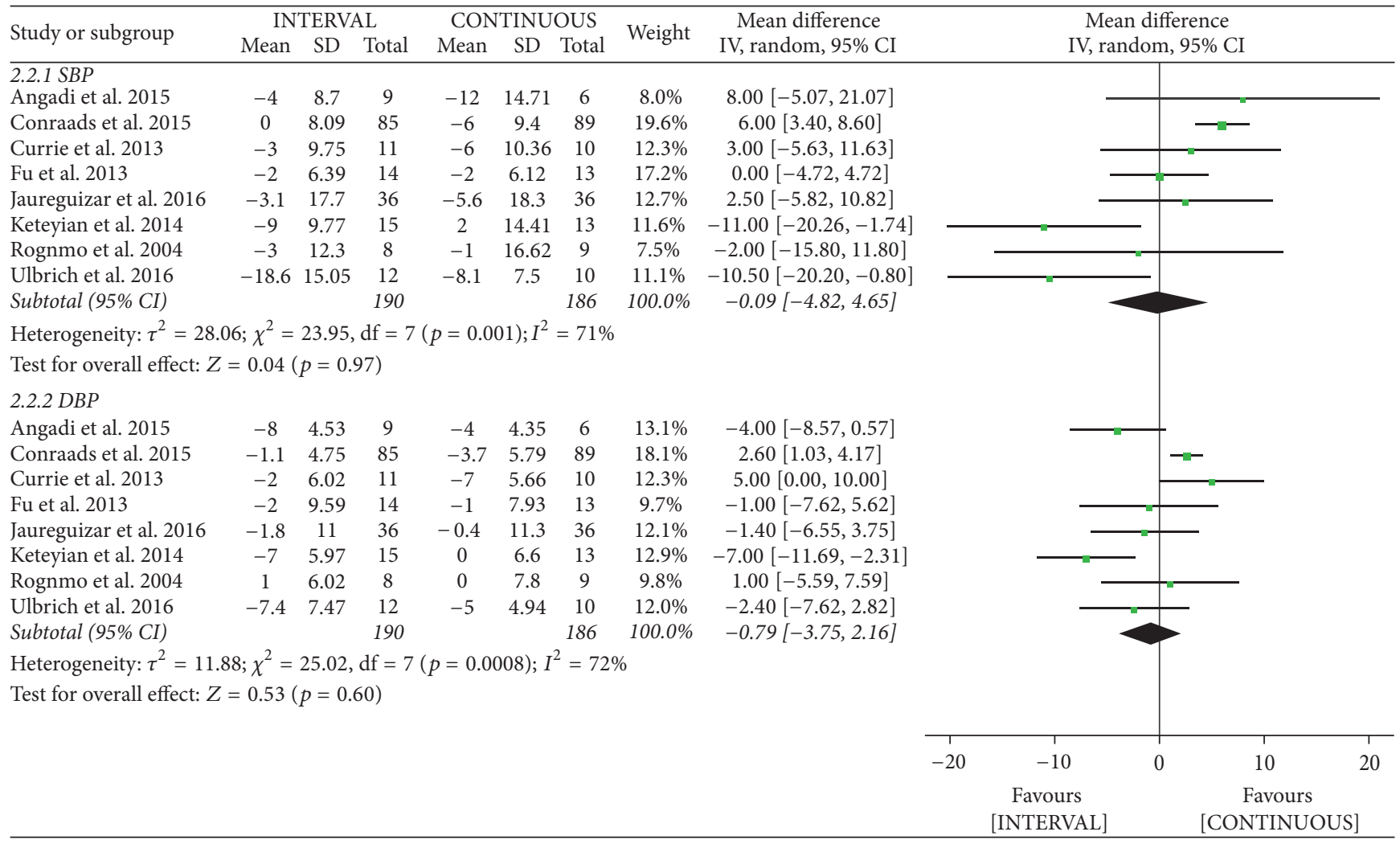

FIGURE 14: Meta-analysis of effects of INTERVAL on blood pressure.

A meta-analysis focused mainly on patients with $\mathrm{CHF}$ by Haykowsky et al. [38] showed INTERVAL is more effective than CONTINUOUS for improving peak $\mathrm{VO}_{2}$ (MD $2.14 \mathrm{~mL} / \mathrm{kg} / \mathrm{min}, 95 \%$ CI 0.66 to $3.63 \mathrm{~mL} / \mathrm{kg} / \mathrm{min}$ ) but not the LVEF. Another systematic analysis by Smart et al. [50] that analyzed $446 \mathrm{CHF}$ patients revealed that INTERVAL determined a significant increase in peak $\mathrm{VO}_{2}$ (MD $1.04 \mathrm{~mL} / \mathrm{kg} / \mathrm{min}, 95 \%$ CI 0.42 to $1.66 \mathrm{~mL} / \mathrm{kg} / \mathrm{min}$ ) and $\mathrm{VE} / \mathrm{VCO}_{2}$ slope ( $\mathrm{MD}-1.35,95 \% \mathrm{CI}-2.15$ to -0.55$)$. A more recent meta-analysis including $\mathrm{CAD}$ patients by Pattyn et al. [51] reported higher increase in VO2 peak with INTERVAL (MD $1.6 \mathrm{~mL} / \mathrm{kg} / \mathrm{min}, 95 \%$ CI 0.8 to $3.02 \mathrm{~mL} / \mathrm{kg} / \mathrm{min}$ ) but $\mathrm{VE} / \mathrm{VCO}_{2}$ slope, $\mathrm{VO} 2$ at $\mathrm{AT}$, and body mass. From our analysis, INTERVAL had similar effect results in improving peak $\mathrm{VO}_{2}$ in above meta-analysis. In addition, our systematic review included more evaluative indicators, such as PHR, peak $\mathrm{V}_{\mathrm{E}}, \mathrm{VE} / \mathrm{VCO}_{2}$ slope, $\mathrm{RER}, \mathrm{VO}_{2}$ at $\mathrm{AT}$, blood pressure, blood lipids, FMD, and LVEF, than did the previous metaanalysis.

Pooled estimates showed significant heterogeneity among studies included in this review. Important clinical and methodological differences may have affected the results obtained in the intervention and control groups. Some of these differences were in inclusion criteria and among participants, who were in different countries and of different ages.
4.2. Study Limitations. Our systematic review has some limitations. Few trials included in the study provided clear descriptions of the randomization and allocation of participants to treatments. Many of the studies failed to describe the blinding of assessors to treatment allocation, which raises the possibility of performance bias. In addition, although we examined publication bias because we searched only four electronic databases, we did not search for unpublished trials. Moreover, the review included only RCTs published in English. Consequently, our results may have been affected by publication bias. Several meta-analyses were affected by statistical heterogeneity, possibly due to differences in study methodologies and data collection techniques (e.g., wide ranges of variability in age, sex, and follow-up duration), which may have affected our findings. Finally, most of the studies had small samples, and no large-scale clinical RCT was included, which likely affected the objectivity and reliability of this meta-analysis and systematic review.

4.3. Conclusion. The current analysis indicated that INTERVAL can provide more benefits than CONTINUOUS in terms of improving peak $\mathrm{VO}_{2}$ and $\mathrm{VO}_{2}$ at $\mathrm{AT}$ in patients with cardiac disease. INTERVAL programs, which increase exercise capacity compared with traditional exercise, are thus preferable. Differences in the effects of INTERVAL and CONTINUOUS in terms of PHR, peak $\mathrm{V}_{\mathrm{E}}, \mathrm{VE} / \mathrm{VCO}_{2}$ slope, RER, body mass, blood pressure, blood lipids, FMD, and 


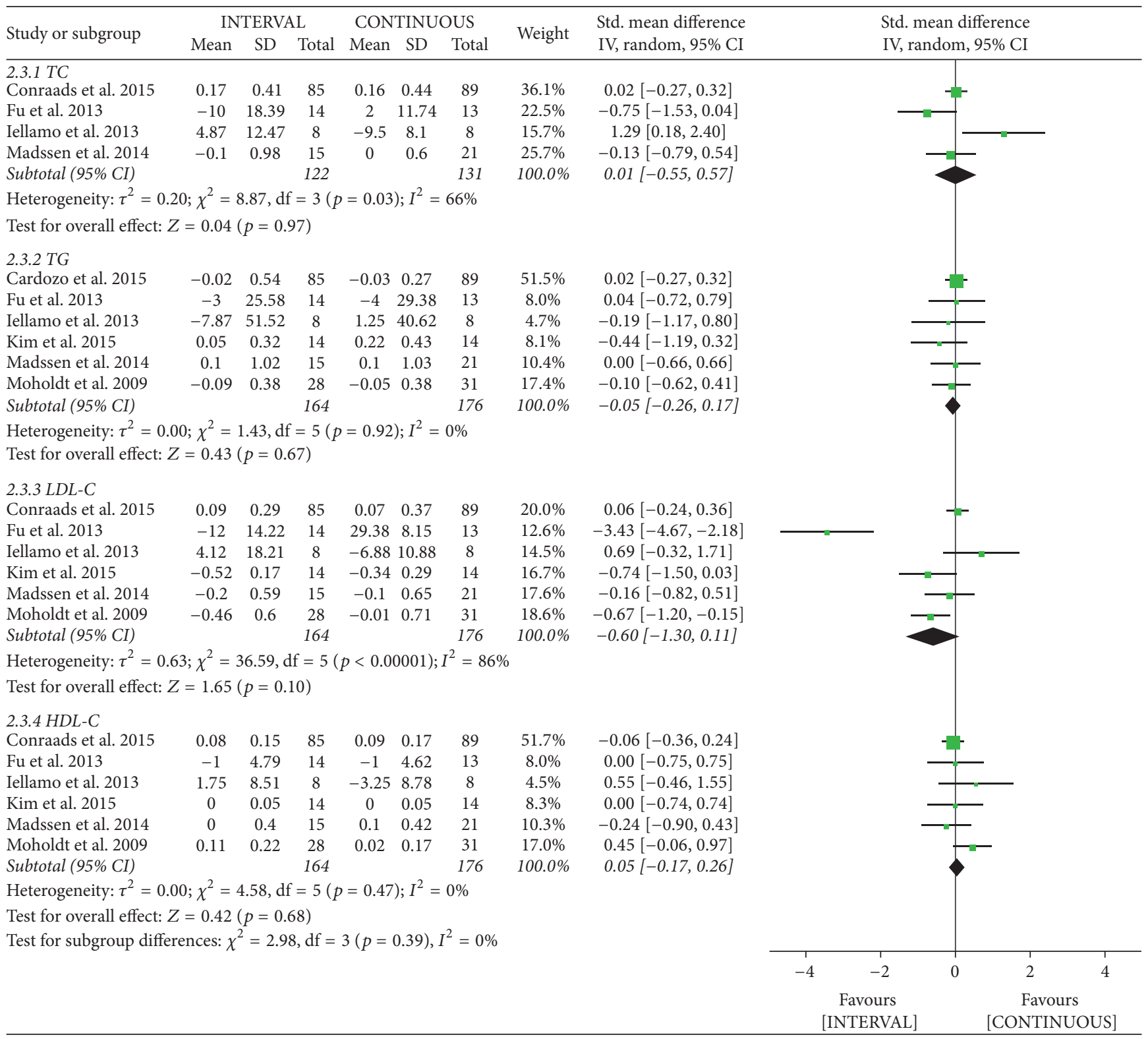

FIGURE 15: Meta-analysis of effects of INTERVAL on blood lipid.

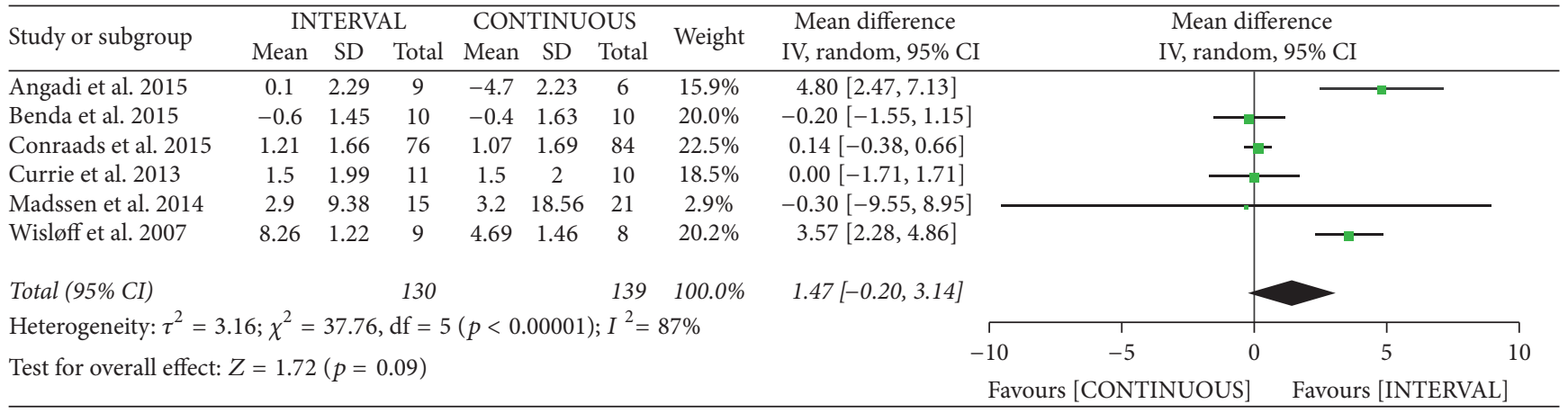




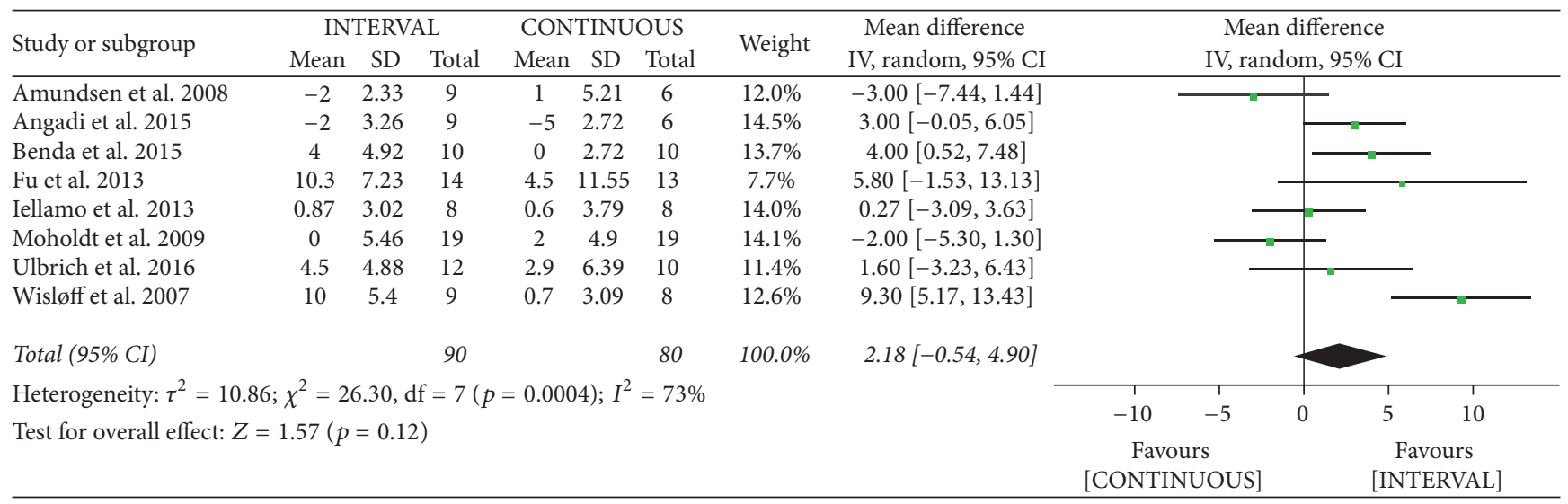

FIGURE 17: Meta-analysis of effects of INTERVAL on LVEF.

LVEF were small and may not be clinically meaningful. The results of this analysis should be interpreted with caution due to the small sample. Accordingly, more high-quality, largesample, multicenter, long-term randomized interventional studies are needed to assess the effects of INTERVAL in cardiac patients.

\section{Appendix}

See Figures 7-17.

\section{Competing Interests}

The authors declare that there are no competing interests regarding the publication of this paper.

\section{References}

[1] S. Mendis, P. Puska, and B. Norrving, Eds., Global Atlas on Cardiovascular Disease Prevention and Control, World Health Organization, Geneva, Switzerland, 2011.

[2] E. M. Antman, D. T. Anbe, P. W. Armstrong et al., "ACC/AHA guidelines for the management of patients with ST-elevation myocardial infarction-executive summary. A report of the American College of Cardiology/American Heart Association Task Force on Practice Guidelines (Writing Committee to revise the 1999 guidelines for the management of patients with acute myocardial infarction)," Journal of the American College of Cardiology, vol. 44, no. 3, pp. 671-719, 2004.

[3] S. A. Hunt, W. T. Abraham, M. H. Chin et al., "ACC/AHA 2005 Guideline Update for the Diagnosis and Management of Chronic Heart Failure in the Adult: a report of the American College of Cardiology/American Heart Association Task Force on Practice Guidelines (Writing Committee to Update the 2001 Guidelines for the Evaluation and Management of Heart Failure): developed in collaboration with the American College of Chest Physicians and the International Society for Heart and Lung Transplantation: endorsed by the Heart Rhythm Society," Circulation, vol. 112, no. 12, pp. e154-e235, 2005.
[4] G. A. Kelley, K. S. Kelley, and Z. Vu Tran, "Aerobic exercise, lipids and lipoproteins in overweight and obese adults: a metaanalysis of randomized controlled trials," International Journal of Obesity, vol. 29, no. 8, pp. 881-893, 2005.

[5] V. A. Cornelissen and R. H. Fagard, "Effect of resistance training on resting blood pressure: a meta-analysis of randomized controlled trials," Journal of Hypertension, vol. 23, no. 2, pp. 251259, 2005.

[6] S. J. Keteyian, C. A. Brawner, P. D. Savage et al., "Peak aerobic capacity predicts prognosis in patients with coronary heart disease," American Heart Journal, vol. 156, no. 2, pp. 292-300, 2008.

[7] H. Brunner, J. R. Cockcroft, J. Deanfield et al., "Endothelial function and dysfunction. Part II: association with cardiovascular risk factors and diseases. A statement by the Working Group on Endothelins and Endothelial Factors of the European Society of Hypertension," Journal of Hypertension, vol. 23, no. 2, pp. 233-246, 2005.

[8] G. F. Fletcher, P. A. Ades, P. Kligfield et al., "Exercise standards for testing and training: a scientific statement from the American heart association," Circulation, vol. 128, no. 8, pp. 873-934, 2013.

[9] A. Z. Ulbrich, V. G. Angarten, A. Schmitt Netto et al., "Comparative effects of high intensity interval training versus moderate intensity continuous training on quality of life in patients with heart failure: study protocol for a randomized controlled trial," Clinical Trials and Regulatory Science in Cardiology, vol. 13, pp. 21-28, 2016.

[10] V. M. Conraads, N. Pattyn, C. De Maeyer et al., "Aerobic interval training and continuous training equally improve aerobic exercise capacity in patients with coronary artery disease: the SAINTEX-CAD study," International Journal of Cardiology, vol. 179, pp. 203-210, 2015.

[11] M. J. Gibala and S. L. McGee, "Metabolic adaptations to shortterm high-intensity interval training: a little pain for a lot of gain?" Exercise and Sport Sciences Reviews, vol. 36, no. 2, pp. 58-63, 2008.

[12] U. Wisløff, A. Støylen, J. P. Loennechen et al., "Superior cardiovascular effect of aerobic interval training versus moderate continuous training in heart failure patients: a randomized study," Circulation, vol. 115, no. 24, pp. 3086-3094, 2007. 
[13] K. D. Currie, J. B. Dubberley, R. S. McKelvie, and M. J. Macdonald, "Low-volume, high-intensity interval training in patients with CAD," Medicine and Science in Sports and Exercise, vol. 45, no. 8, pp. 1436-1442, 2013.

[14] S. Ito, T. Mizoguchi, and T. Saeki, "Review of high-intensity interval training in cardiac rehabilitation," Internal Medicine, vol. 55, no. 17, pp. 2329-2336, 2016.

[15] F. Giallauria, N. A. Smart, A. Cittadini, and C. Vigorito, "Exercise training modalities in chronic heart failure: does high intensity aerobic interval training make the difference?" Monaldi Archives for Chest Disease, vol. 86, no. 1-2, 2016.

[16] A. D. Elliott, K. Rajopadhyaya, D. J. Bentley, J. F. Beltrame, and E. C. Aromataris, "Interval training versus continuous exercise in patients with coronary artery disease: a meta-analysis," Heart Lung and Circulation, vol. 24, no. 2, pp. 149-157, 2015.

[17] J. P. T. Higgins and S. Green, Cochrane Handbook for Systematic Reviews of Interventions Version 5.1.0, The Cochrane Collaboration, 2011, http://handbook.cochrane.org/.

[18] "The George Institute for Global Health and the University of Sydney. Physiotherapy Evidence Database," 2012, http://www .pedro.org.au/english/downloads/pedro-scale/.

[19] B. H. Amundsen, Ø. Rognmo, G. Hatlen-Rebhan, and S. A. Slørdahl, "High-intensity aerobic exercise improves diastolic function in coronary artery disease," Scandinavian Cardiovascular Journal, vol. 42, no. 2, pp. 110-117, 2008.

[20] S. S. Angadi, F. Mookadam, C. D. Lee, W. J. Tucker, M. J. Haykowsky, and G. A. Gaesser, "High-intensity interval training vs. moderate-intensity continuous exercise training in heart failure with preserved ejection fraction: a pilot study," Journal of Applied Physiology, vol. 119, no. 6, pp. 753-758, 2015.

[21] N. M. Benda, J. P. Seeger, G. G. Stevens et al., "Effects of high-intensity interval training versus continuous training on physical fitness, cardiovascular function and quality of life in heart failure patients," PLOS ONE, vol. 10, no. 10, 2015.

[22] G. G. Cardozo, R. B. Oliveira, and P. T. V. Farinatti, "Effects of high intensity interval versus moderate continuous training on markers of ventilatory and cardiac efficiency in coronary heart disease patients," The Scientific World Journal, vol. 2015, Article ID 192479, 8 pages, 2015.

[23] S. Dimopoulos, M. Anastasiou-Nana, D. Sakellariou et al., "Effects of exercise rehabilitation program on heart rate recovery in patients with chronic heart failure," European Journal of Cardiovascular Prevention \& Rehabilitation, vol. 13, no. 1, pp. 6773, 2006.

[24] C. Freyssin, C. Verkindt, F. Prieur, P. Benaich, S. Maunier, and P. Blanc, "Cardiac rehabilitation in chronic heart failure: effect of an 8-week, high-intensity interval training versus continuous training," Archives of Physical Medicine and Rehabilitation, vol. 93, no. 8, pp. 1359-1364, 2012.

[25] T.-C. Fu, C.-H. Wang, P.-S. Lin et al., "Aerobic interval training improves oxygen uptake efficiency by enhancing cerebral and muscular hemodynamics in patients with heart failure," International Journal of Cardiology, vol. 167, no. 1, pp. 41-50, 2013.

[26] F. Iellamo, V. Manzi, G. Caminiti et al., "Matched dose interval and continuous exercise training induce similar cardiorespiratory and metabolic adaptations in patients with heart failure," International Journal of Cardiology, vol. 167, no. 6, pp. 25612565, 2013.

[27] K. V. Jaureguizar, D. Vicente-Campos, L. R. Bautista et al., "Effect of high-intensity interval versus continuous exercise training on functional capacity and quality of life in patients with coronary artery disease: a randomized clinical trial," Journal of Cardiopulmonary Rehabilitation and Prevention, vol. 36, no. 2, pp. 96-105, 2016.

[28] S. J. Keteyian, B. A. Hibner, K. Bronsteen et al., "Greater improvement in cardiorespiratory fitness using higher-intensity interval training in the standard cardiac rehabilitation setting," Journal of Cardiopulmonary Rehabilitation and Prevention, vol. 34, no. 2, pp. 98-105, 2014.

[29] C. Kim, H. E. Choi, and M. H. Lim, "Effect of high interval training in acute myocardial infarction patients with drug-eluting stent," American Journal of Physical Medicine and Rehabilitation, vol. 94, no. 10, pp. 879-886, 2015.

[30] P. Koufaki, T. H. Mercer, K. P. George, and J. Nolan, "Lowvolume high-intensity interval training vs continuous aerobic cycling in patients with chronic heart failure: a pragmatic randomised clinical trial of feasibility and effectiveness," Journal of Rehabilitation Medicine, vol. 46, no. 4, pp. 348-356, 2014.

[31] E. Madssen, T. Moholdt, V. Videm, U. Wisløff, K. Hegbom, and R. Wiseth, "Coronary atheroma regression and plaque characteristics assessed by grayscale and radiofrequency intravascular ultrasound after aerobic exercise," American Journal of Cardiology, vol. 114, no. 10, pp. 1504-1511, 2014.

[32] T. T. Moholdt, B. H. Amundsen, L. A. Rustad et al., "Aerobic interval training versus continuous moderate exercise after coronary artery bypass surgery: a randomized study of cardiovascular effects and quality of life," American Heart Journal, vol. 158, no. 6, pp. 1031-1037, 2009.

[33] D. Prado, E. Rocco, A. Silva et al., "Effects of continuous vs interval exercise training on oxygen uptake efficiency slope in patients with coronary artery disease," Brazilian Journal of Medical and Biological Research, vol. 49, no. 2, Article ID e4890, 2016.

[34] E. A. Rocco, D. M. L. Prado, A. G. Silva et al., "Effect of continuous and interval exercise training on the PETCO2 response during a graded exercise test in patients with coronary artery disease," Clinics, vol. 67, no. 6, pp. 623-627, 2012.

[35] P. Roditis, S. Dimopoulos, D. Sakellariou et al., "The effects of exercise training on the kinetics of oxygen uptake in patients with chronic heart failure," European Journal of Cardiovascular Prevention \& Rehabilitation, vol. 14, no. 2, pp. 304-311, 2007.

[36] Ø. Rognmo, E. Hetland, J. Helgerud, J. Hoff, and S. A. Slørdahl, "High intensity aerobic interval exercise is superior to moderate intensity exercise for increasing aerobic capacity in patients with coronary artery disease," European Journal of Cardiovascular Prevention and Rehabilitation, vol. 11, no. 3, pp. 216-222, 2004.

[37] D. E. R. Warburton, D. C. McKenzie, M. J. Haykowsky et al., "Effectiveness of high-intensity interval training for the rehabilitation of patients with coronary artery disease," American Journal of Cardiology, vol. 95, no. 9, pp. 1080-1084, 2005.

[38] M. J. Haykowsky, M. P. Timmons, C. Kruger, M. McNeely, D. A. Taylor, and A. M. Clark, "Meta-analysis of aerobic interval training on exercise capacity and systolic function in patients with heart failure and reduced ejection fractions," American Journal of Cardiology, vol. 111, no. 10, pp. 1466-1469, 2013.

[39] H. A. Wenger and G. J. Bell, "The interactions of intensity, frequency and duration of exercise training in altering cardiorespiratory fitness," Sports Medicine, vol. 3, no. 5, pp. 346356, 1986.

[40] U. Wisløff, Ø. Ellingsen, and O. J. Kemi, "High-intensity interval training to maximize cardiac benefits of exercise training?" 
Exercise and Sport Sciences Reviews, vol. 37, no. 3, pp. 139-146, 2009.

[41] D. Hoshino, Y. Yoshida, Y. Kitaoka, H. Hatta, and A. Bonen, "High-intensity interval training increases intrinsic rates of mitochondrial fatty acid oxidation in rat red and white skeletal muscle," Applied Physiology, Nutrition, and Metabolism, vol. 38, no. 3, pp. 326-333, 2013.

[42] A. E. Tjønna, S. J. Lee, Ø. Rognmo et al., "Aerobic interval training versus continuous moderate exercise as a treatment for the metabolic syndrome: A Pilot Study," Circulation, vol. 118, no. 4, pp. 346-354, 2008.

[43] M. J. Gibala, J. P. Little, M. J. Macdonald, and J. A. Hawley, "Physiological adaptations to low-volume, high-intensity interval training in health and disease," Journal of Physiology, vol. 590, no. 5, pp. 1077-1084, 2012.

[44] A. S. Leon, B. A. Franklin, F. Costa et al., "Cardiac rehabilitation and secondary prevention of coronary heart disease: an American Heart Association scientific statement from the Council on Clinical Cardiology (Subcommittee on Exercise, Cardiac Rehabilitation, and Prevention) and the Council on Nutrition, Physical Activity, and Metabolism (Subcommittee on Physical Activity), in collaboration with the American association of Cardiovascular and Pulmonary Rehabilitation," Circulation, vol. 111, no. 3, pp. 369-376, 2005.

[45] P. A. Ades, N. M. Green, and C. E. Coello, "Effects of exercise and cardiac rehabilitation on cardiovascular outcomes," Cardiology Clinics, vol. 21, no. 3, pp. 435-448, 2003.

[46] C. J. Lavie, R. J. Thomas, R. W. Squires, T. G. Allison, and R. V. Milani, "Exercise training and cardiac rehabilitation in primary and secondary prevention of coronary heart disease," Mayo Clinic Proceedings, vol. 84, no. 4, pp. 373-383, 2009.

[47] F. Roveda, H. R. Middlekauff, M. U. P. B. Rondon et al., "The effects of exercise training on sympathetic neural activation in advanced heart failure: a randomized controlled trial," Journal of the American College of Cardiology, vol. 42, no. 5, pp. 854-860, 2003.

[48] T. Guiraud, A. Nigam, V. Gremeaux, P. Meyer, M. Juneau, and L. Bosquet, "High-intensity interval training in cardiac rehabilitation," Sports Medicine, vol. 42, no. 7, pp. 587-605, 2012.

[49] N. Oldridge, "Exercise-based cardiac rehabilitation in patients with coronary heart disease: meta-analysis outcomes revisited," Future Cardiology, vol. 8, no. 5, pp. 729-751, 2012.

[50] N. A. Smart, G. Dieberg, and F. Giallauria, "Intermittent versus continuous exercise training in chronic heart failure: a metaanalysis," International Journal of Cardiology, vol. 166, no. 2, pp. 352-358, 2013.

[51] N. Pattyn, E. Coeckelberghs, R. Buys, V. A. Cornelissen, and L. Vanhees, "Aerobic interval training vs. moderate continuous training in coronary artery disease patients: a systematic review and meta-analysis," Sports Medicine, vol. 44, no. 5, pp. 687-700, 2014. 

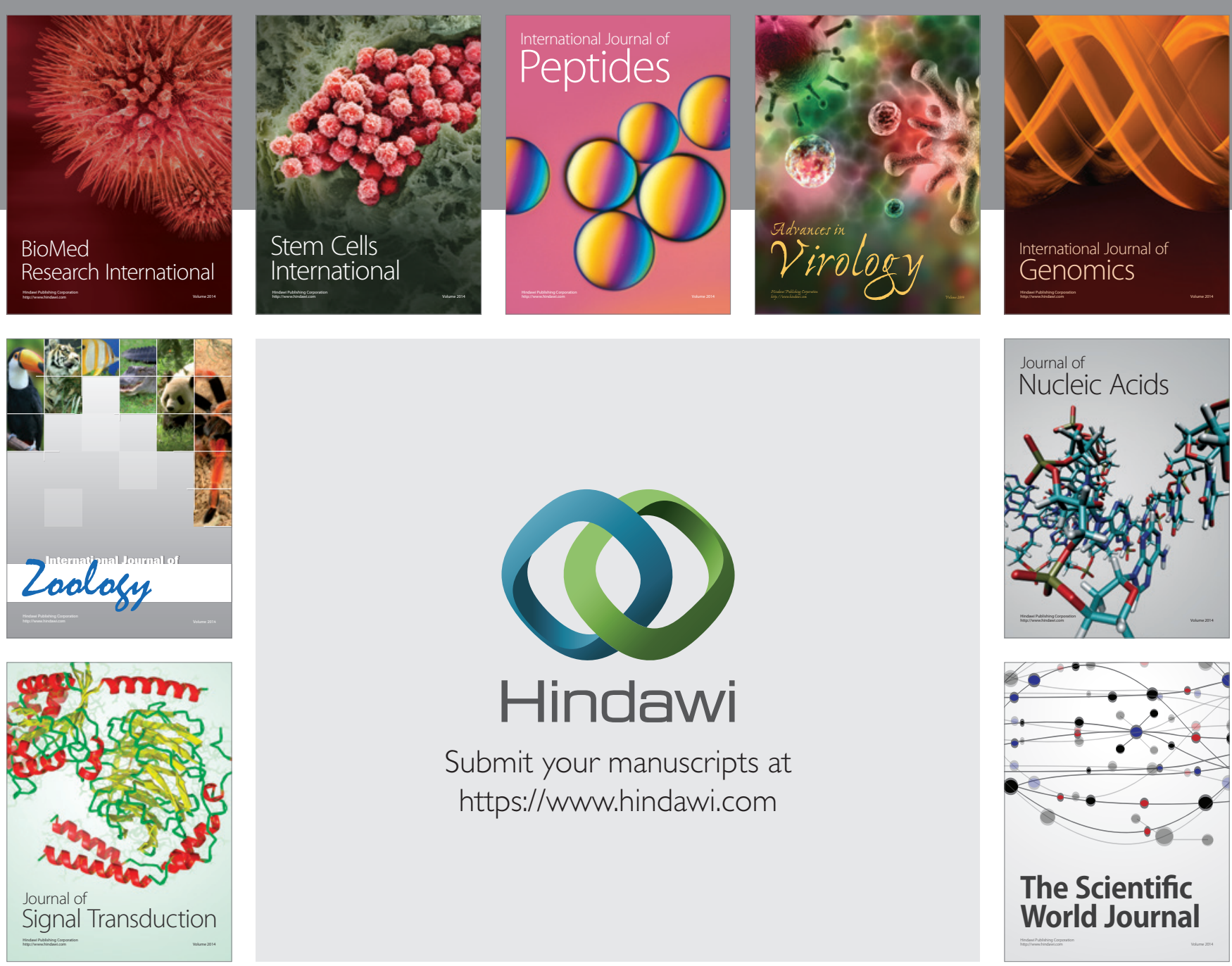

Submit your manuscripts at

https://www.hindawi.com
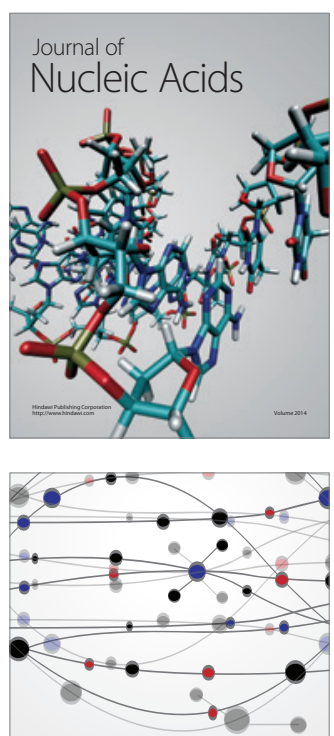

The Scientific World Journal
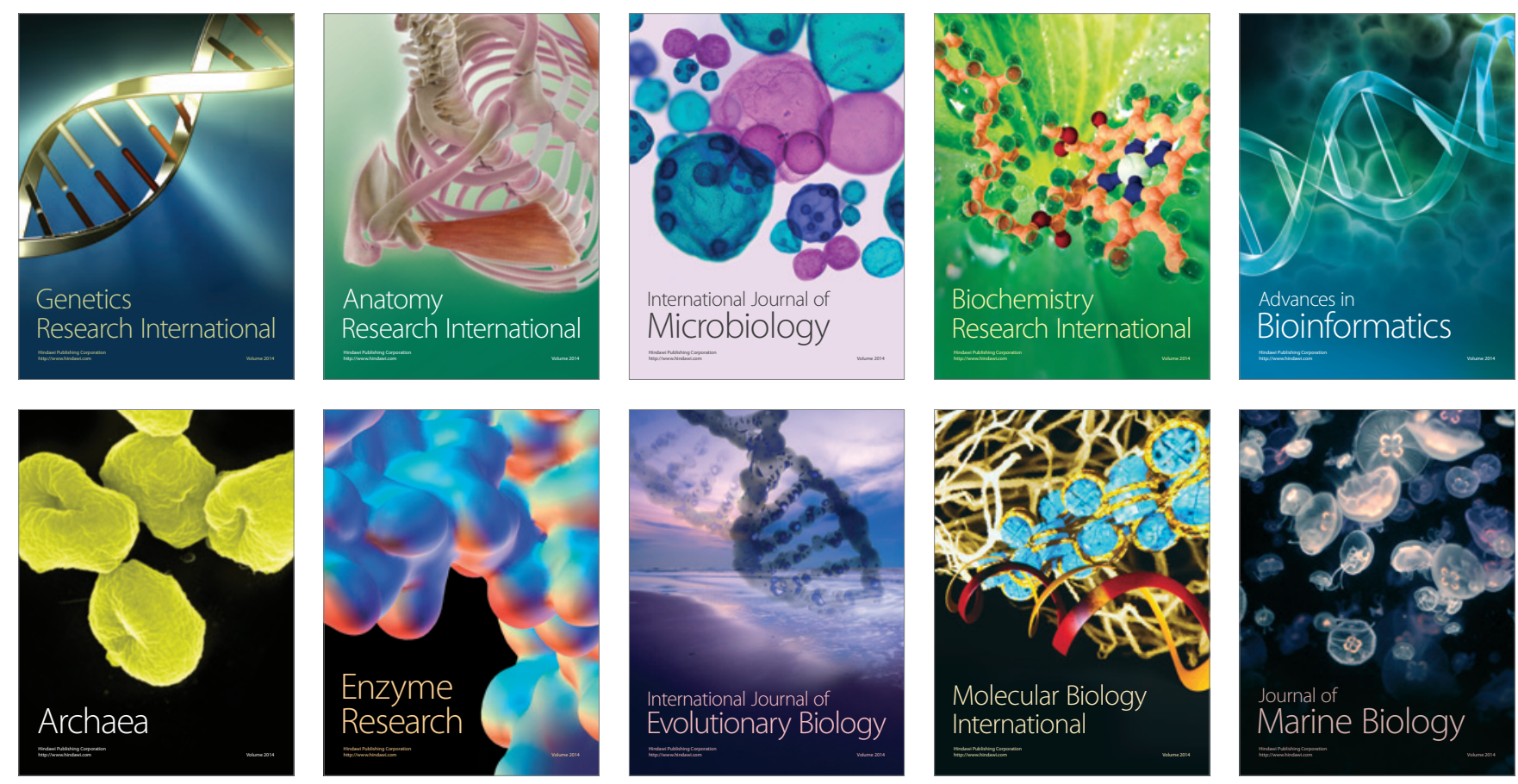